\title{
Molecular dynamics simulation of amorphous poly(3-hexylthiophene)
}

\author{
Flora D. Tsourtou, ${ }^{1}$ Loukas D. Peristeras, ${ }^{2}$ Rossen Apostolov ${ }^{3}$ and Vlasis G. Mavrantzas ${ }^{1,4}$
}

1. Department of Chemical Engineering, University of Patras, GR 26504, Patras, Greece \&

FORTH-ICE/HT, GR 26504, Patras, Greece

2. National Center for Scientific Research "Demokritos", Institute of Nanoscience and Nanotechnology, Molecular Thermodynamics and Modelling of Materials Laboratory, GR-15310 Aghia Paraskevi Attikis, Greece

3. KTH Royal Institute of Technology, SE-100 44 Stockholm, Sweden 4. ETH Zürich, Department of Mechanical and Process Engineering, Particle Technology Laboratory, CH8092 Zürich, Switzerland

\section{Electronic Supplementary Information (ESI)}

The electronic supplementary information (ESI) file consists of three Sections. Section 1 presents the functional form and the parameter values of the seven force fields (FFs) considered in this work for the MD simulation of amorphous regioregular P3HT (or RR-P3HT) using the GROMACS ${ }^{1}$ software. Section 2 describes differences in the predictions of these FFs for certain relaxational, structural, conformational, and thermodynamic properties of the simulated systems with some more emphasis on the distribution of the dihedral angles and of the radial pair correlation functions of thiophene rings' centers-of-mass. Theoretical predictions from the Worm Like Chain (WLC) model and values of the density at $300 \mathrm{~K}$ from extrapolation are also considered. Section 3 presents additional results from the MD simulations with the force field proposed by Poelking and Andrienko ${ }^{2}$ on the correlation of the persistence length with the conjugation length, the distribution of the major dihedral angles as a function 
of temperature, the spatial extent of cis and trans segments, and the estimation of the ECL based on unweighted and weighted distributions.

S1. Force fields implemented in this work and their parameterization

\section{S1.1 FF-1 by Cheung et al. ${ }^{3}$}

The functional form of FF-1 is

$$
\begin{aligned}
& U=\sum_{\text {bonds }} \frac{k_{r}}{2}\left(r-r_{0}\right)^{2}+\sum_{\text {angles }} \frac{k_{\theta}}{2}\left(\theta-\theta_{0}\right)^{2}+\sum_{\text {dihedrals }} \sum_{n=1}^{m} k_{n}\left(1+\cos \left(n \phi-\phi_{n}\right)\right) \\
& +\sum_{\text {impropers }} \sum_{n=1}^{m} k_{n}\left(1+\cos \left(n \phi-\phi_{n}\right)\right)+ \\
& \sum_{\text {nonbonded }}\left\{4 \varepsilon_{i j}\left[\left(\frac{\sigma_{i j}}{r_{i j}}\right)^{12}-\left(\frac{\sigma_{i j}}{r_{i j}}\right)^{6}\right]+\frac{C q_{i} q_{j}}{\varepsilon_{r} r_{i j}}\right\}
\end{aligned}
$$

The coding of atom types for RR-P3HT and the values of the force field parameters for the non-bonded and bonded interactions in FF-1 are presented in Table S1.1. 
Table S1.1. Potential form and parameters of FF-1.

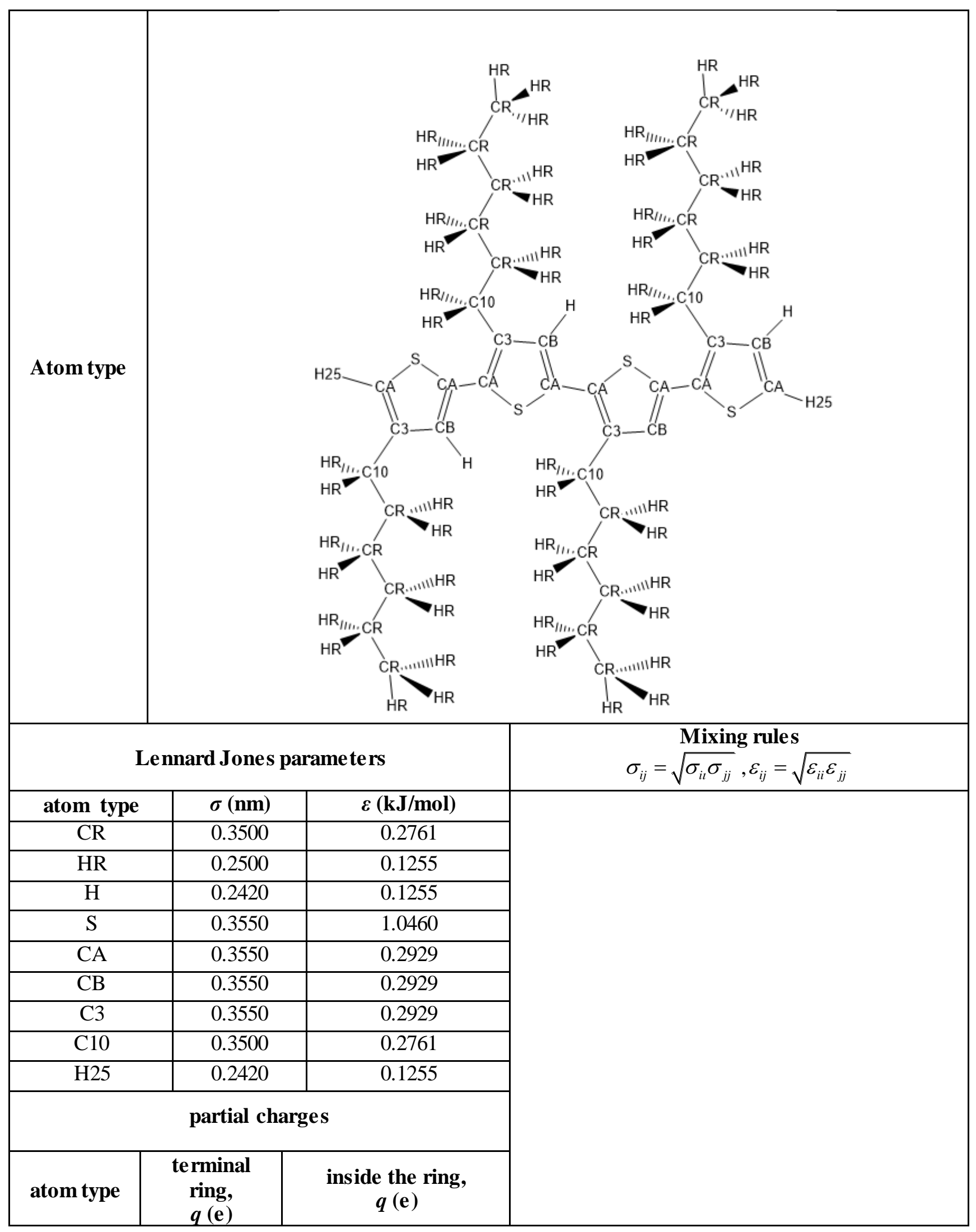




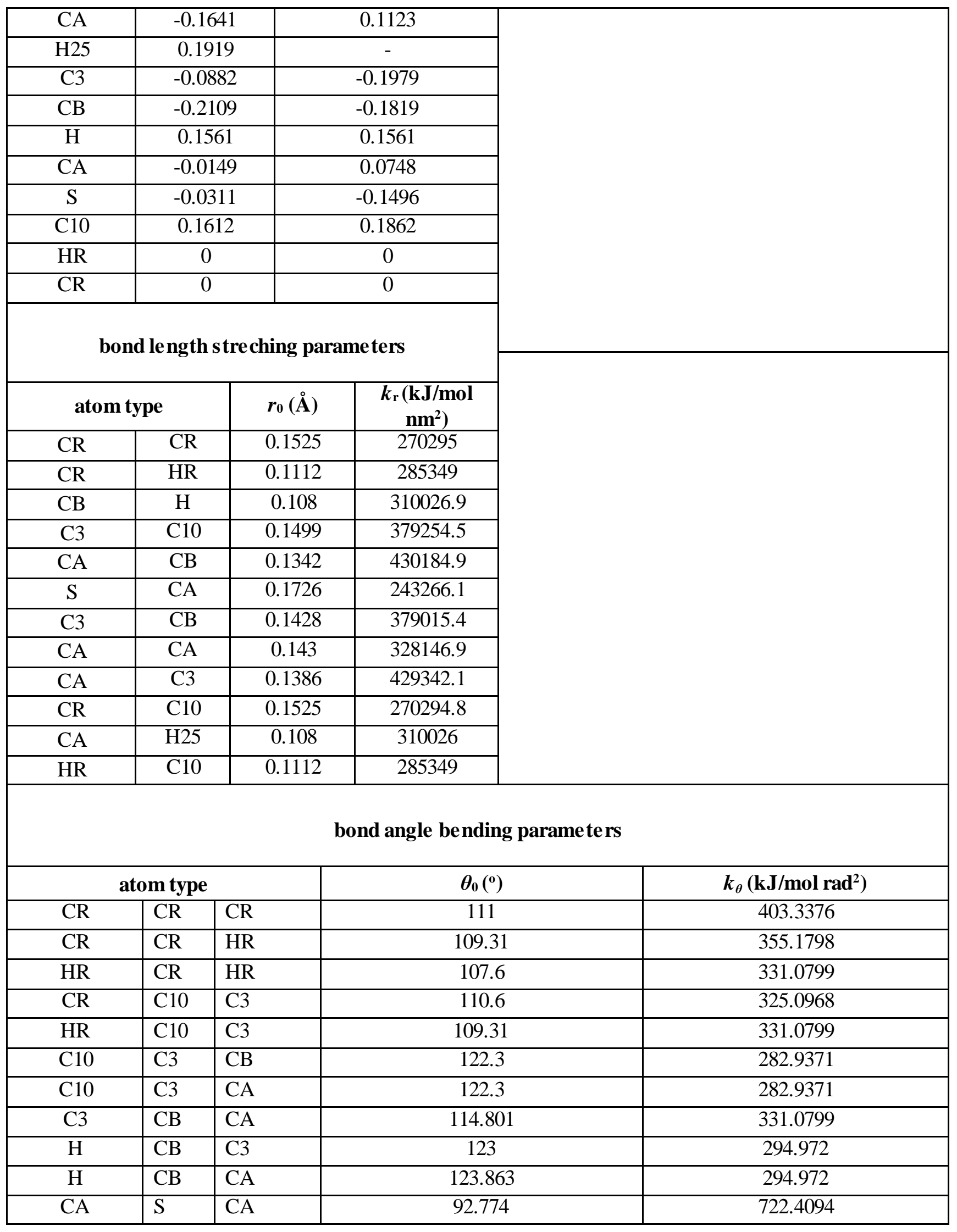




\begin{tabular}{|c|c|c|c|c|c|c|}
\hline$\overline{\mathrm{CA}}$ & $\mathrm{CA}$ & \multicolumn{2}{|l|}{ C3 } & \multicolumn{2}{|c|}{127.505} & 457.5204 \\
\hline $\mathrm{S}$ & CA & $\mathrm{CA}$ & & \multicolumn{2}{|c|}{119.569} & 349.1548 \\
\hline S & $\mathrm{CA}$ & \multicolumn{2}{|l|}{ CB } & \multicolumn{2}{|c|}{109.871} & 722.4094 \\
\hline $\mathrm{H} 2$ & CA & \multicolumn{2}{|l|}{$\mathrm{S}$} & \multicolumn{2}{|c|}{123} & 240.7892 \\
\hline$\overline{\mathrm{H} 2}$ & $\mathrm{CA}$ & \multicolumn{2}{|l|}{$\mathrm{CB}$} & \multicolumn{2}{|c|}{125.1} & 294.972 \\
\hline$\overline{\mathrm{CA}}$ & $\mathrm{CA}$ & \multicolumn{2}{|l|}{$\mathrm{CB}$} & \multicolumn{2}{|c|}{130.554} & 457.5204 \\
\hline$\overline{\mathrm{C} 3}$ & $\mathrm{CA}$ & \multicolumn{2}{|l|}{$\mathrm{S}$} & \multicolumn{2}{|c|}{110.658} & 722.4094 \\
\hline$\overline{\mathrm{H} 2}$ & $\mathrm{CA}$ & \multicolumn{2}{|l|}{$\mathrm{C} 3$} & \multicolumn{2}{|c|}{125.1} & 294.972 \\
\hline$\overline{\mathrm{CA}}$ & $\mathrm{C} 3$ & \multicolumn{2}{|l|}{ CB } & \multicolumn{2}{|c|}{111.873} & 331.0799 \\
\hline $\mathrm{C} 1$ & $\mathrm{CR}$ & $\mathrm{HR}$ & & & & 355.1798 \\
\hline $\mathrm{CR}$ & $\mathrm{C} 10$ & HR & & & & 355.1798 \\
\hline $\mathrm{HR}$ & $\mathrm{C} 10$ & $\mathrm{HR}$ & & & & 331.0799 \\
\hline $\mathrm{CR}$ & $\begin{array}{l}\mathrm{CR} \\
\end{array}$ & $\mathrm{C} 10$ & & & & 403.3376 \\
\hline & & & & ihedra & & \\
\hline & atom ty & & & $\phi_{n}\left({ }^{\circ}\right)$ & $k_{n}(\mathrm{~kJ} / \mathrm{mol})$ & $n$ \\
\hline $\mathrm{CR}$ & $\mathrm{CR}$ & CR & CR & 0 & 3.64008 & 1 \\
\hline & & & & -180 & -0.328444 & $\frac{2}{2}$ \\
\hline & & & & 0 & 0.665256 & 3 \\
\hline $\mathrm{CR}$ & $\mathrm{CR}$ & CR & HR & 0 & 0.765672 & 3 \\
\hline HR & $\mathrm{CR}$ & $\mathrm{CR}$ & $\mathrm{HR}$ & 0 & 0.665256 & 3 \\
\hline HR & CR & $\mathrm{C} 10$ & C3 & 0 & 0.966504 & 3 \\
\hline $\mathrm{CR}$ & $\mathrm{CR}$ & C10 & $\mathrm{C} 3$ & 0 & 0 & 1 \\
\hline HR & C10 & $\mathrm{C} 3$ & $\mathrm{CB}$ & 0 & 0 & 1 \\
\hline HR & $\mathrm{C} 10$ & $\mathrm{C} 3$ & CA & 0 & 0 & 1 \\
\hline CR & C10 & $\mathrm{C} 3$ & $\mathrm{CB}$ & 0 & 0 & 1 \\
\hline CA & $\mathrm{C} 3$ & $\mathrm{C} 10$ & CR & 0 & 0 & 1 \\
\hline $\bar{S}$ & $\mathrm{CA}$ & $\mathrm{CA}$ & $\mathrm{C} 3$ & 0 & 0 & 1 \\
\hline $\mathrm{CB}$ & $\mathrm{CA}$ & CA & C3 & 0 & 0 & 1 \\
\hline$S$ & $\mathrm{CA}$ & $\mathrm{CA}$ & $S$ & 0 & -0.79496 & 1 \\
\hline & & & & 0 & -2.99156 & 2 \\
\hline & & & & 0 & -0.0356 & 3 \\
\hline & & & & 0 & 1.933008 & 4 \\
\hline $\mathrm{CA}$ & $\mathrm{CB}$ & $\mathrm{C} 3$ & CA & -180 & 19.89492 & 2 \\
\hline $\mathrm{H}$ & $\mathrm{CB}$ & $\mathrm{C} 3$ & $\mathrm{CA}$ & -180 & 19.89492 & 2 \\
\hline $\mathrm{H}$ & $\mathrm{CB}$ & C3 & $\mathrm{C} 10$ & -180 & 19.89492 & 2 \\
\hline $\mathrm{CA}$ & $\mathrm{CB}$ & $\mathrm{C} 3$ & C10 & -180 & 19.89492 & 2 \\
\hline $\mathrm{CB}$ & $\mathrm{CA}$ & $\mathrm{CA}$ & $\mathrm{S}$ & 0 & 0 & 1 \\
\hline $\mathrm{C} 10$ & $\mathrm{CR}$ & CR & CR & 0 & 3.64008 & 1 \\
\hline & & & & & -0.328444 & $\overline{2}$ \\
\hline & & & & & 0.665256 & 3 \\
\hline HR & C10 & $\mathrm{CR}$ & CR & 0 & 0.765672 & 3 \\
\hline
\end{tabular}




\begin{tabular}{|c|c|c|c|c|c|c|}
\hline HR & C10 & CR & HR & 0 & 0.665256 & 3 \\
\hline S & CA & C3 & C10 & -180 & 19.89492 & 2 \\
\hline CA & CA & C3 & C10 & -180 & 19.89492 & 2 \\
\hline S & CA & CB & C3 & -180 & 19.89492 & 2 \\
\hline S & CA & C3 & CB & -180 & 19.89492 & 2 \\
\hline CA & S & CA & CA & -180 & 19.89492 & 2 \\
\hline CA & S & CA & CB & -180 & 19.89492 & 2 \\
\hline C3 & CB & CA & CA & -180 & 19.89492 & 2 \\
\hline C3 & CA & S & CA & -180 & 19.89492 & 2 \\
\hline CA & CA & C3 & CB & -180 & 19.89492 & 2 \\
\hline C10 & CR & CR & HR & 0 & 0.765672 & 2 \\
\hline H & CB & CA & CA & -180 & 19.89492 & 2 \\
\hline H & CB & CA & S & -180 & 19.89492 & 2 \\
\hline H25 & CA & S & CA & -180 & 19.89492 & 2 \\
\hline H25 & CA & C3 & CB & -180 & 19.89492 & 2 \\
\hline H25 & CA & C3 & C10 & -180 & 19.89492 & 2 \\
\hline H25 & CA & CB & C3 & -180 & 19.89492 & 2 \\
\hline H25 & CA & CB & H & -180 & 19.89492 & 2.2048 \\
\hline
\end{tabular}




\section{S1.2 FF-2 by Huang et al. ${ }^{4}$}

The functional form of FF-2 is

$$
\begin{aligned}
& U=\sum_{\text {bonds }} \frac{k_{r}}{2}\left(r-r_{0}\right)^{2}+\sum_{\text {angles }} \frac{k_{\theta}}{2}\left(\theta-\theta_{0}\right)^{2}+\sum_{\text {dihedrals }} \sum_{n=1}^{m} k_{n}\left(1+\cos \left(n \phi+\phi_{n}\right)\right)+ \\
& \sum_{\text {nonbonded }}\left\{4 \varepsilon_{i j}\left[\left(\frac{\sigma_{i j}}{r_{i j}}\right)^{12}-\left(\frac{\sigma_{i j}}{r_{i j}}\right)^{6}\right]+\frac{C q_{i} q_{j}}{\varepsilon_{r} r_{i j}}\right\}
\end{aligned}
$$

The coding of atom types for RR- P3HT and the values of the force field parameters for the non-bonded and bonded interactions in FF-2 are presented in Table S1.2. 
Table S1.2. Potential from and parameters of FF-2.

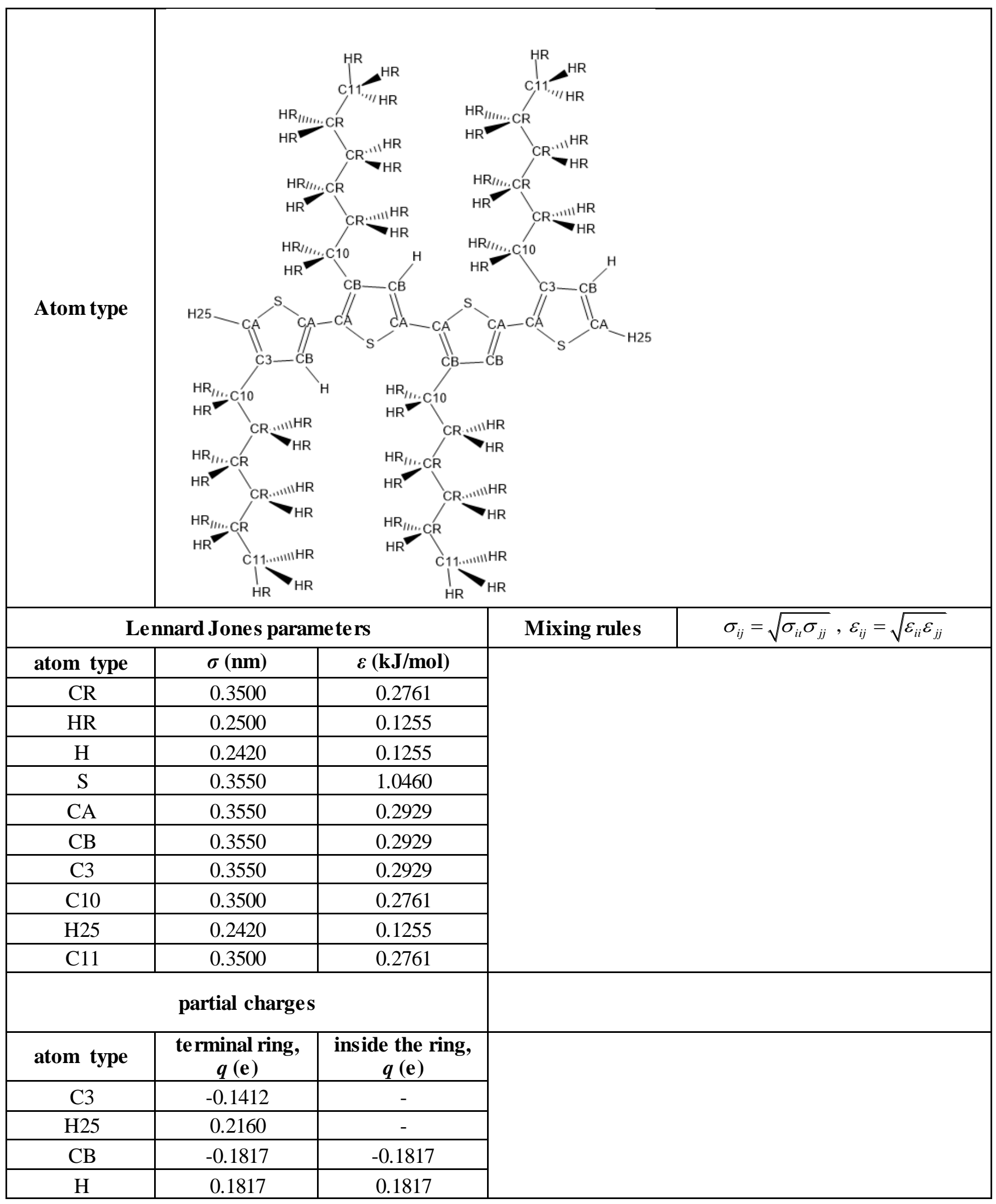




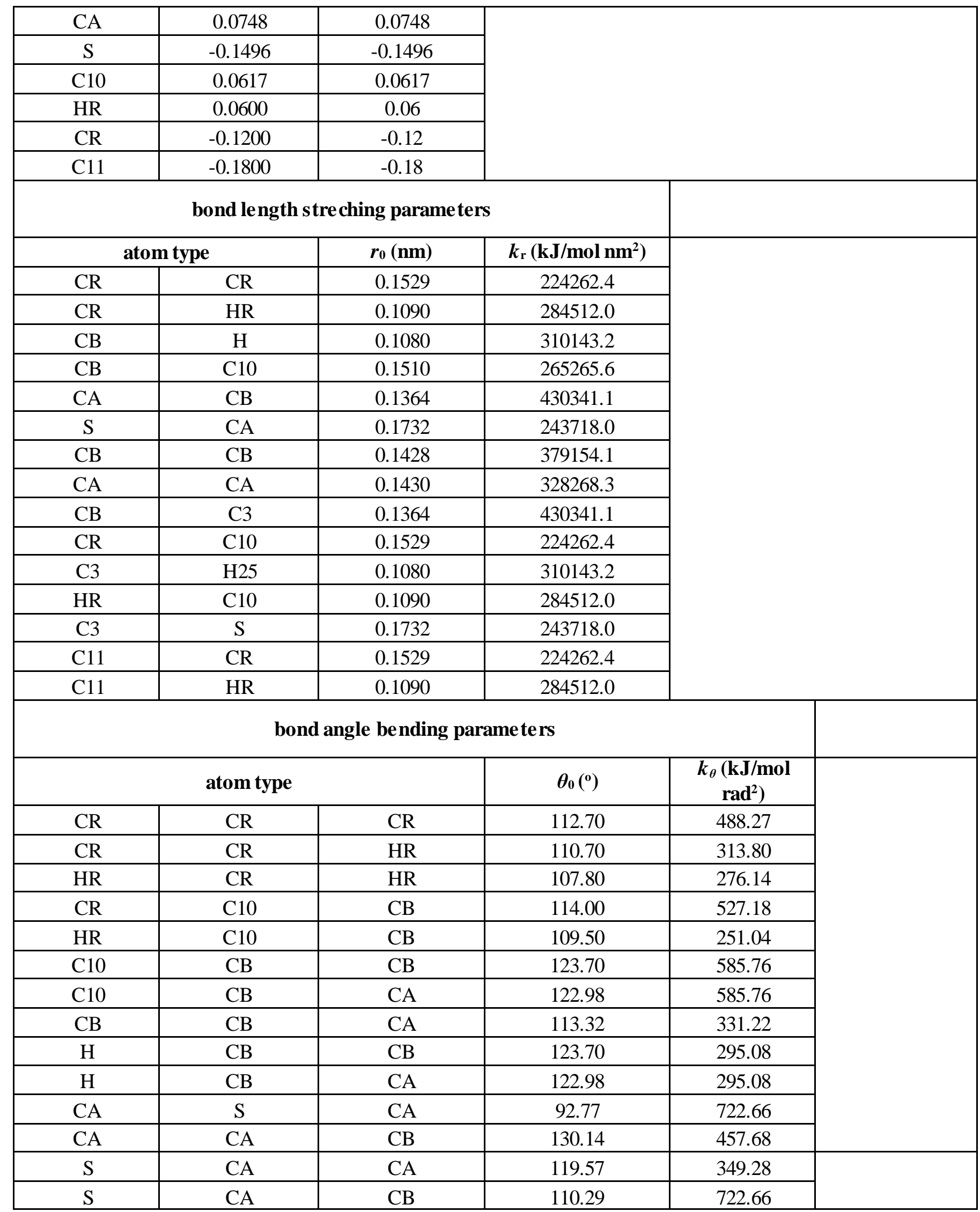




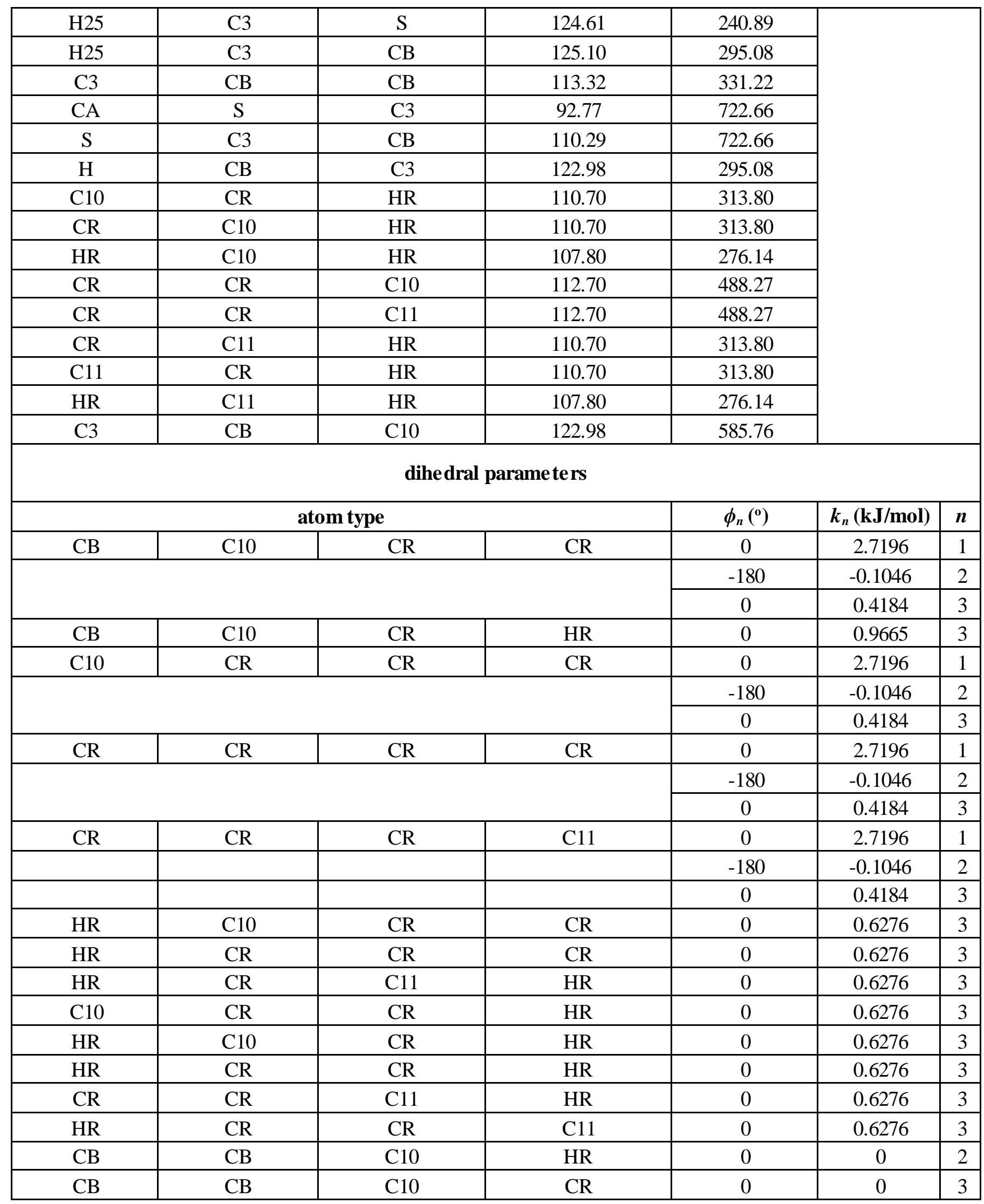




\begin{tabular}{|c|c|c|c|c|c|c|}
\hline $\mathrm{CA}$ & $\mathrm{CB}$ & $\mathrm{C} 10$ & $\mathrm{HR}$ & 0 & 0 & 3 \\
\hline $\mathrm{C} 3$ & $\mathrm{CB}$ & $\mathrm{C} 10$ & HR & 0 & 0 & 3 \\
\hline CA & $\mathrm{CB}$ & $\mathrm{C} 10$ & $\mathrm{CR}$ & 0 & 0 & 3 \\
\hline C3 & $\mathrm{CB}$ & $\mathrm{C} 10$ & $\mathrm{CR}$ & 0 & 0 & 3 \\
\hline $\mathrm{CB}$ & $\mathrm{CA}$ & $\mathrm{CA}$ & $\mathrm{S}$ & 0 & 0 & 3 \\
\hline $\mathrm{CB}$ & CA & CA & CB & 0 & 0 & 3 \\
\hline \multirow[t]{15}{*}{$\mathrm{S}$} & $\mathrm{CA}$ & $\mathrm{CA}$ & $\mathrm{S}$ & 0 & -0.5221632 & 1 \\
\hline & & & & 180 & 11.1464689 & 2 \\
\hline & & & & 0 & 0.1124659 & 3 \\
\hline & & & & 180 & -0.3603679 & 4 \\
\hline & & & & 0 & $\begin{array}{l}0.5319956 \\
\end{array}$ & 5 \\
\hline & & & & 180 & 0.1810835 & 6 \\
\hline & & & & 0 & 0.2702446 & 7 \\
\hline & & & & 180 & 0.0266521 & 8 \\
\hline & & & & 0 & 0.0018428 & 9 \\
\hline & & & & 180 & 0.001889 & 10 \\
\hline & & & & 0 & 0.0019397 & 11 \\
\hline & & & & 180 & 0.0019942 & 12 \\
\hline & & & & 0 & 0.0020529 & 13 \\
\hline & & & & 180 & 0.0021149 & 14 \\
\hline & & & & 0 & 0.0021805 & 15 \\
\hline $\mathrm{C} 3$ & $\mathrm{~S}$ & $\mathrm{CA}$ & $\mathrm{CB}$ & -180 & 0 & 2 \\
\hline $\mathrm{C} 3$ & $\mathrm{CB}$ & $\mathrm{CB}$ & $\mathrm{CA}$ & 0 & 0 & 1 \\
\hline \multirow{3}{*}{$\mathrm{CB}$} & & & & -180 & 0 & 2 \\
\hline & $\mathrm{CB}$ & $\mathrm{CA}$ & $\mathrm{S}$ & -180 & 0 & 2 \\
\hline & & & & 0 & 0 & 3 \\
\hline $\mathrm{CB}$ & $\mathrm{C} 3$ & $S$ & $\mathrm{CA}$ & -180 & 0 & 2 \\
\hline \multirow[t]{2}{*}{$\mathrm{CB}$} & $\mathrm{CB}$ & $\mathrm{C} 3$ & $\mathrm{~S}$ & -180 & 0 & 2 \\
\hline & & & & 0 & 0 & 3 \\
\hline \multirow[t]{2}{*}{$\mathrm{CA}$} & $\mathrm{CB}$ & $\mathrm{CB}$ & $\mathrm{CA}$ & 0 & 0 & 1 \\
\hline & & & & -180 & 0 & 2 \\
\hline $\mathrm{CA}$ & $\mathrm{S}$ & $\mathrm{CA}$ & $\mathrm{CB}$ & -180 & 0 & 2 \\
\hline \multirow[t]{3}{*}{$\mathrm{CA}$} & $\mathrm{CB}$ & $\mathrm{CB}$ & $\mathrm{H}$ & 0 & 0 & 1 \\
\hline & & & & -180 & 0 & 2 \\
\hline & & & & 0 & 0 & 3 \\
\hline \multirow[t]{3}{*}{$\mathrm{C} 3$} & $\mathrm{CB}$ & $\mathrm{CB}$ & $\mathrm{H}$ & 0 & 0 & 1 \\
\hline & & & & -180 & 0 & 2 \\
\hline & & & & 0 & 0 & 3 \\
\hline \multirow[t]{2}{*}{$\mathrm{S}$} & $\mathrm{C} 3$ & $\mathrm{CB}$ & $\mathrm{H}$ & 0 & 0 & 1 \\
\hline & & & & -180 & 0 & 2 \\
\hline $\mathrm{H} 25$ & $\mathrm{C} 3$ & CB & $\mathrm{H}$ & -180 & 0 & 2 \\
\hline
\end{tabular}




\begin{tabular}{|c|c|c|c|c|c|c|}
\hline $\mathrm{CA}$ & $S$ & $\mathrm{C} 3$ & $\mathrm{H} 25$ & -180 & 0 & 2 \\
\hline $\mathrm{H} 25$ & $\mathrm{C} 3$ & $\mathrm{CB}$ & $\mathrm{CB}$ & 0 & 0 & 1 \\
\hline & & & & -180 & 0 & 2 \\
\hline & & & & 0 & 0 & 3 \\
\hline$S$ & $\mathrm{CA}$ & $\mathrm{CB}$ & $\mathrm{H}$ & 0 & 0 & 1 \\
\hline & & & & -180 & 0 & 2 \\
\hline $\mathrm{CA}$ & $\mathrm{CA}$ & $S$ & $\mathrm{CA}$ & -180 & 0 & 2 \\
\hline & & & & 0 & 0 & 3 \\
\hline $\mathrm{CB}$ & $\mathrm{CB}$ & $\mathrm{CA}$ & $\mathrm{CA}$ & 0 & 0 & 1 \\
\hline & & & & -180 & 0 & 2 \\
\hline C3 & $S$ & $\mathrm{CA}$ & $\mathrm{CA}$ & -180 & 0 & 2 \\
\hline & & & & 0 & 0 & 3 \\
\hline $\mathrm{CA}$ & $\mathrm{CA}$ & $\mathrm{CB}$ & $\mathrm{H}$ & 0 & 0 & 1 \\
\hline & & & & -180 & 0 & 2 \\
\hline & & & & 0 & 0 & 3 \\
\hline $\mathrm{CA}$ & $\mathrm{CA}$ & $\mathrm{CB}$ & $\mathrm{C} 10$ & 0 & 0 & 1 \\
\hline & & & & -180 & 0 & 2 \\
\hline & & & & 0 & 0 & 3 \\
\hline C3 & $\mathrm{CB}$ & $\mathrm{CB}$ & $\mathrm{C} 10$ & 0 & 0 & 1 \\
\hline & & & & -180 & 0 & 2 \\
\hline & & & & 0 & 0 & 3 \\
\hline $\mathrm{S}$ & $\mathrm{CA}$ & $\mathrm{CB}$ & $\mathrm{C} 10$ & 0 & 0 & 1 \\
\hline & & & & -180 & 0 & 2 \\
\hline $\mathrm{H}$ & $\mathrm{CB}$ & $\mathrm{CB}$ & $\mathrm{C} 10$ & -180 & 0 & 2 \\
\hline $\mathrm{CA}$ & $\mathrm{CB}$ & $\mathrm{CB}$ & $\mathrm{C} 10$ & 0 & 0 & 1 \\
\hline & & & & -180 & 0 & 2 \\
\hline & & & & 0 & 0 & 3 \\
\hline$S$ & C3 & $\mathrm{CB}$ & $\mathrm{C} 10$ & 0 & 0 & 1 \\
\hline & & & & -180 & 0 & 2 \\
\hline $\mathrm{H} 25$ & $\mathrm{C} 3$ & $\mathrm{CB}$ & $\mathrm{C} 10$ & -180 & 0 & 2 \\
\hline
\end{tabular}




\section{S1.3 FF-3 by Scwharz et al. 5}

The functional form of FF-3 is

$$
\begin{aligned}
& U=\sum_{\text {bonds }} \frac{k_{r}}{2}\left(r-r_{0}\right)^{2}+\sum_{\text {angles }} \frac{k_{\theta}}{2}\left(\theta-\theta_{0}\right)^{2}+\sum_{\text {dihedrals }} \sum_{n=1}^{m} k_{n}\left(1+\cos \left(n \phi-\phi_{n}\right)\right) \\
& +\sum_{\text {impropers }} \sum_{n=1}^{m} k_{n}\left(1+\cos \left(n \phi-\phi_{n}\right)\right)+ \\
& \sum_{\text {nonbonded }}\left\{4 \varepsilon_{i j}\left[\left(\frac{\sigma_{i j}}{r_{i j}}\right)^{12}-\left(\frac{\sigma_{i j}}{r_{i j}}\right)^{6}\right]+\frac{C q_{i} q_{j}}{\varepsilon_{r} r_{i j}}\right\}
\end{aligned}
$$

The coding of atom types for RR-P3HT and the values of the force field parameters for the non-bonded and bonded interactions in FF-3 are presented in Table S1.3. 
Table S1.3. Potential form and parameters of FF-3.

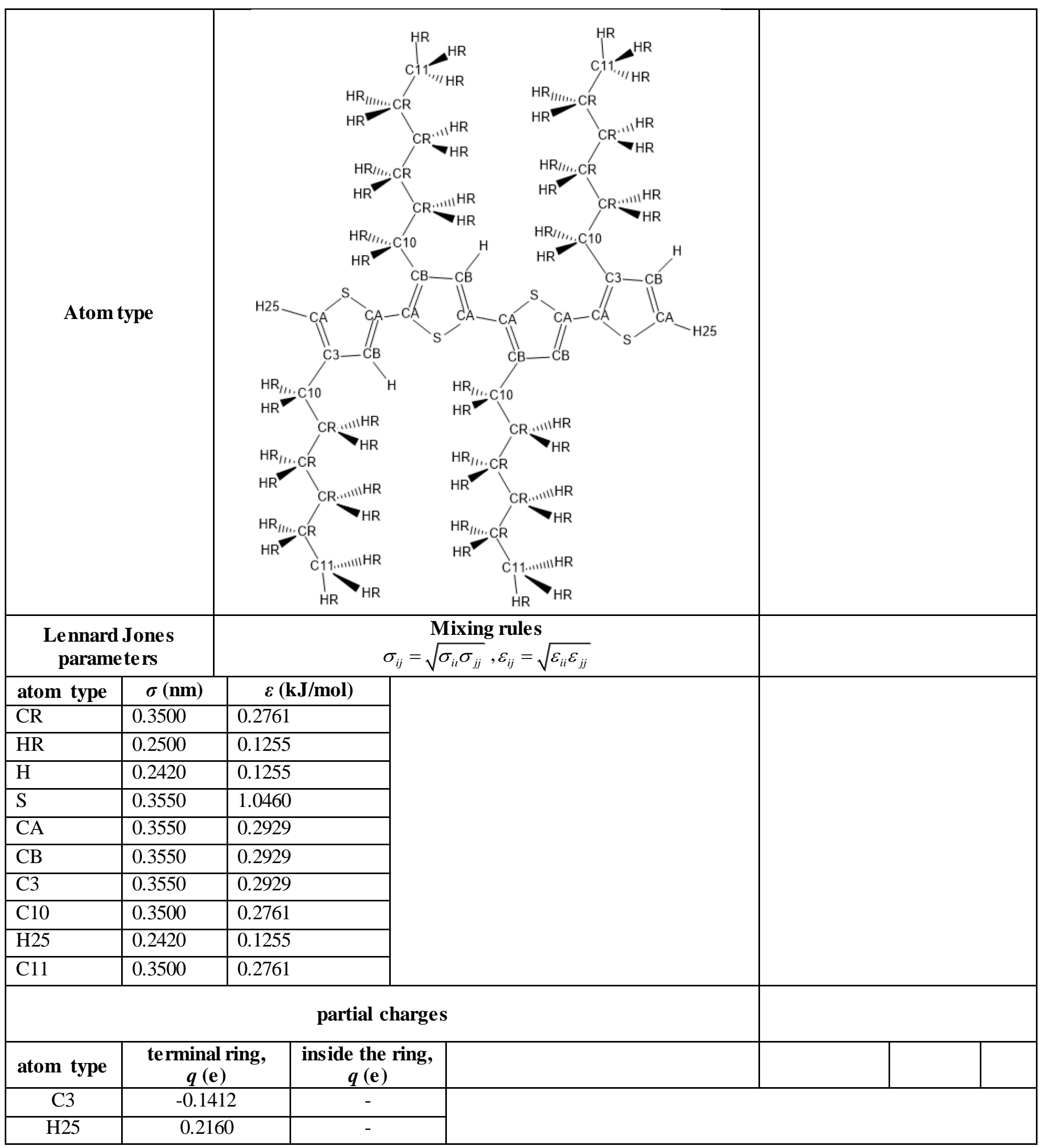




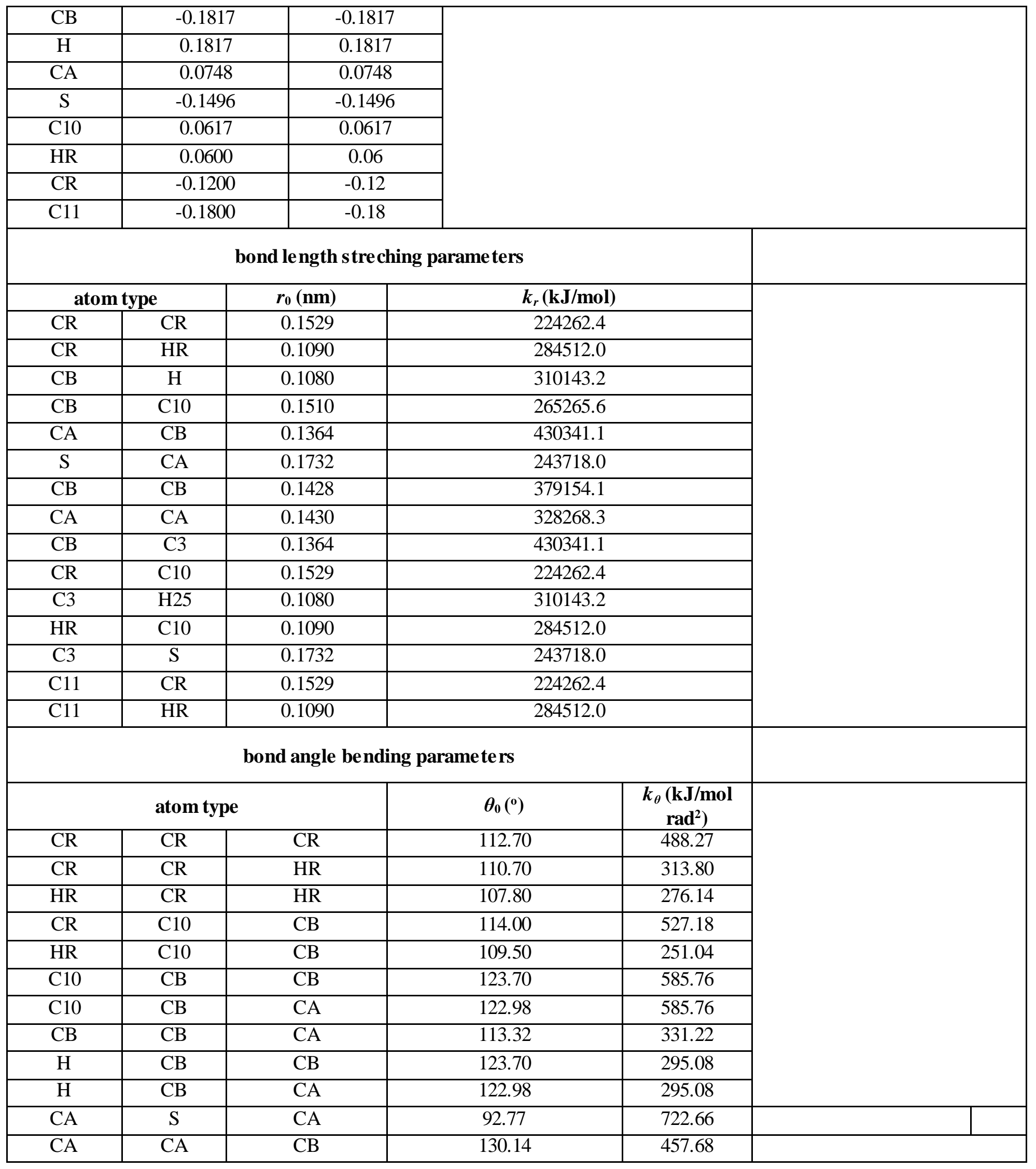




\begin{tabular}{|c|c|c|c|c|}
\hline S & CA & CA & 119.57 & 349.28 \\
\hline S & CA & CB & 110.29 & 722.66 \\
\hline H25 & C3 & S & 124.61 & 240.89 \\
\hline H25 & C3 & CB & 125.10 & 295.08 \\
\hline C3 & CB & CB & 113.32 & 331.22 \\
\hline CA & S & C3 & 92.77 & 722.66 \\
\hline S & C3 & CB & 110.29 & 722.66 \\
\hline H & CB & C3 & 122.98 & 295.08 \\
\hline C10 & CR & HR & 110.70 & 313.80 \\
\hline CR & C10 & HR & 110.70 & 313.80 \\
\hline HR & C10 & HR & 107.80 & 276.14 \\
\hline CR & CR & C10 & 112.70 & 488.27 \\
\hline CR & CR & C11 & 112.70 & 488.27 \\
\hline CR & C11 & HR & 110.70 & 313.80 \\
\hline C11 & CR & HR & 110.70 & 313.80 \\
\hline HR & C11 & HR & 107.80 & 276.14 \\
\hline C3 & CB & C10 & 122.98 & 585.76 \\
\hline
\end{tabular}

dihedral parameters

\begin{tabular}{|c|c|c|c|c|c|c|}
\hline \multicolumn{4}{|c|}{ atom type } & $\phi_{n}\left({ }^{0}\right)$ & $k_{n}(\mathrm{~kJ} / \mathrm{mol})$ & $\mathbf{n}$ \\
\hline CB & C10 & $\mathrm{CR}$ & CR & 0 & 2.7196 & 1 \\
\hline $\mathrm{CB}$ & C10 & CR & CR & -180 & -0.1046 & 2 \\
\hline $\mathrm{CB}$ & $\mathrm{C} 10$ & CR & CR & 0 & 0.4184 & 3 \\
\hline CB & $\mathrm{C} 10$ & CR & HR & 0 & 0.9665 & 3 \\
\hline $\mathrm{C} 10$ & $\mathrm{CR}$ & CR & CR & 0 & 2.7196 & 1 \\
\hline C10 & $\mathrm{CR}$ & CR & CR & -180 & -0.1046 & 2 \\
\hline $\mathrm{C} 10$ & $\mathrm{CR}$ & CR & CR & 0 & 0.4184 & 3 \\
\hline $\mathrm{CR}$ & $\mathrm{CR}$ & CR & CR & 0 & 2.7196 & 1 \\
\hline CR & $\mathrm{CR}$ & CR & CR & -180 & -0.1046 & 2 \\
\hline $\mathrm{CR}$ & $\overline{\mathrm{CR}}$ & $\overline{C R}$ & CR & 0 & 0.4184 & 3 \\
\hline $\mathrm{CR}$ & $\mathrm{CR}$ & $\overline{\mathrm{CR}}$ & $\overline{\mathrm{C} 11}$ & 0 & 2.7196 & 1 \\
\hline $\mathrm{CR}$ & $\mathrm{CR}$ & CR & $\mathrm{C} 11$ & -180 & -0.1046 & 2 \\
\hline $\mathrm{CR}$ & $\mathrm{CR}$ & CR & $\bar{C} 11$ & 0 & 0.4184 & 3 \\
\hline HR & C10 & CR & CR & 0 & 0.6276 & 3 \\
\hline HR & $\mathrm{CR}$ & CR & CR & 0 & 0.6276 & 3 \\
\hline $\mathrm{HR}$ & $\mathrm{CR}$ & C11 & HR & 0 & 0.6276 & 3 \\
\hline C10 & $\mathrm{CR}$ & CR & HR & 0 & 0.6276 & 3 \\
\hline
\end{tabular}




\begin{tabular}{|c|c|c|c|c|c|c|}
\hline $\mathrm{HR}$ & $\mathrm{C} 10$ & CR & $\mathrm{HR}$ & 0 & 0.6276 & 3 \\
\hline $\mathrm{HR}$ & $\mathrm{CR}$ & $\mathrm{CR}$ & HR & 0 & 0.6276 & 3 \\
\hline $\mathrm{CR}$ & CR & $\mathrm{C} 11$ & HR & 0 & 0.6276 & 3 \\
\hline $\mathrm{HR}$ & $\mathrm{CR}$ & $\mathrm{CR}$ & C11 & 0 & 0.6276 & 3 \\
\hline & & & & & & \\
\hline $\mathrm{CB}$ & $\mathrm{CB}$ & $\mathrm{C} 10$ & HR & 0 & 0 & 2 \\
\hline $\mathrm{CB}$ & $\mathrm{CB}$ & $\mathrm{C} 10$ & CR & 0 & 0 & 3 \\
\hline $\mathrm{CA}$ & $\mathrm{CB}$ & $\mathrm{C} 10$ & $\mathrm{HR}$ & 0 & 0 & 3 \\
\hline $\mathrm{C} 3$ & $\mathrm{CB}$ & $\mathrm{C} 10$ & HR & 0 & 0 & 3 \\
\hline $\mathrm{CA}$ & $\mathrm{CB}$ & $\mathrm{C} 10$ & CR & 0 & 0 & 3 \\
\hline $\mathrm{C} 3$ & $\mathrm{CB}$ & $\mathrm{C} 10$ & $\mathrm{CR}$ & 0 & 0 & 3 \\
\hline $\mathrm{CB}^{\mathrm{C}}$ & CA & CA & $\mathrm{S}$ & -180 & -051296 & 0 \\
\hline $\mathrm{CB}$ & $\mathrm{CA}$ & CA & $\mathrm{S}$ & 0 & 0.300071 & 1 \\
\hline $\mathrm{CB}$ & $\mathrm{CA}$ & $\mathrm{CA}$ & $\mathrm{S}$ & -180 & 3.544515 & 2 \\
\hline $\mathrm{CB}$ & $\mathrm{CA}$ & $\mathrm{CA}$ & $\mathrm{S}$ & $\overline{0}$ & 0.036898 & 3 \\
\hline $\mathrm{CB}$ & $\mathrm{CA}$ & $\mathrm{CA}$ & $\mathrm{S}$ & -180 & 0.156233 & 4 \\
\hline $\mathrm{CB}$ & $\mathrm{CA}$ & $\mathrm{CA}$ & $\mathrm{S}$ & 0 & -0.00866 & 5 \\
\hline $\mathrm{CB}$ & $\mathrm{CA}$ & $\mathrm{CA}$ & $\mathrm{S}$ & -180 & 0.110209 & 6 \\
\hline $\mathrm{CB}$ & $\mathrm{CA}$ & $\mathrm{CA}$ & $\mathrm{S}$ & 0 & -0.07183 & 7 \\
\hline $\mathrm{CB}$ & $\mathrm{CA}$ & $\mathrm{CA}$ & $\mathrm{S}$ & -180 & 0.144207 & 8 \\
\hline $\mathrm{CB}$ & $\mathrm{CA}$ & $\mathrm{CA}$ & $\mathrm{CB}$ & -180 & 0 & 0 \\
\hline $\mathrm{CB}$ & $\mathrm{CA}$ & $\mathrm{CA}$ & $\mathrm{CB}$ & 0 & -0.30007 & 1 \\
\hline$\overline{C B}$ & $\mathrm{CA}$ & $\mathrm{CA}$ & $\mathrm{CB}$ & -180 & 3.544515 & 2 \\
\hline $\mathrm{CB}$ & $\mathrm{CA}$ & $\mathrm{CA}$ & $\mathrm{CB}$ & 0 & -0.0369 & 3 \\
\hline $\mathrm{CB}$ & $\mathrm{CA}$ & $\mathrm{CA}$ & $\mathrm{CB}$ & -180 & 0.156233 & 4 \\
\hline $\mathrm{CB}$ & CA & CA & $\mathrm{CB}$ & 0 & 0.008656 & 5 \\
\hline CB & $\mathrm{CA}$ & $\mathrm{CA}$ & $\mathrm{CB}$ & -180 & 0.110209 & 6 \\
\hline $\mathrm{CB}$ & $\mathrm{CA}$ & $\mathrm{CA}$ & $\mathrm{CB}$ & 0 & 0.071834 & 7 \\
\hline $\mathrm{CB}$ & $\mathrm{CA}$ & $\mathrm{CA}$ & $\mathrm{CB}$ & -180 & 0.144207 & 8 \\
\hline $\mathrm{S}_{1}$ & $S_{A}$ & $S_{A}$ & $\mathrm{~S}_{\mathrm{S}}$ & -180 & 0 & 0 \\
\hline $\bar{S}$ & CA & $\mathrm{CA}$ & $\mathrm{S}$ & 0 & -0.30007 & 1 \\
\hline $\mathrm{S}$ & $\mathrm{CA}$ & $\mathrm{CA}$ & $\mathrm{S}$ & -180 & 3.544515 & 2 \\
\hline $\mathrm{S}$ & $\mathrm{CA}$ & $\mathrm{CA}$ & $\mathrm{S}$ & 0 & -0.0369 & 3 \\
\hline $\mathrm{S}$ & $\mathrm{CA}$ & $\mathrm{CA}$ & $\mathrm{S}$ & -180 & 0.156233 & 4 \\
\hline $\mathrm{S}$ & $\mathrm{CA}$ & $\mathrm{CA}$ & $\mathrm{S}$ & 0 & 0.008656 & 5 \\
\hline $\mathrm{S}$ & $\mathrm{CA}$ & $\mathrm{CA}$ & $\mathrm{S}$ & -180 & 0.110209 & 6 \\
\hline $\bar{S}$ & $\mathrm{CA}$ & $\mathrm{CA}$ & $\mathrm{S}$ & 0 & 0.071834 & 7 \\
\hline $\mathrm{S}$ & $\mathrm{CA}$ & $\mathrm{CA}$ & $\mathrm{S}$ & -180 & 0.144207 & 8 \\
\hline
\end{tabular}




\begin{tabular}{|c|c|c|c|c|c|c|}
\hline $\mathrm{C} 3$ & $S$ & $\mathrm{CA}$ & $\mathrm{CB}$ & -180 & 15.167 & 2 \\
\hline C3 & $\mathrm{CB}$ & $\mathrm{CB}$ & CA & -180 & 15.167 & 2 \\
\hline $\mathrm{CB}$ & $\mathrm{CB}$ & $\mathrm{CA}$ & $S$ & -180 & 15.167 & 2 \\
\hline $\mathrm{CB}$ & $\mathrm{C} 3$ & $\mathrm{~S}$ & $\mathrm{CA}$ & -180 & 15.167 & 2 \\
\hline$\overline{C B}$ & $\overline{C B}$ & $\mathrm{C} 3$ & $\mathrm{~S}$ & -180 & 15.167 & 2 \\
\hline CA & $\mathrm{CB}$ & CB & $\mathrm{CA}$ & -180 & 15.167 & 2 \\
\hline & & & & & & \\
\hline CA & $\mathrm{S}$ & $\mathrm{CA}$ & CB & -180 & 15.167 & 2 \\
\hline CA & CB & CB & $\mathrm{H}$ & -180 & 15.167 & 2 \\
\hline C3 & CB & $\mathrm{CB}$ & $\mathrm{H}$ & -180 & 15.167 & 2 \\
\hline $\mathrm{S}$ & C3 & $\mathrm{CB}$ & $\overline{\mathrm{H}}$ & -180 & 15.167 & 2 \\
\hline $\mathrm{H} 25$ & C3 & $\overline{C B}$ & $\mathrm{H}$ & -180 & 15.167 & 2 \\
\hline CA & $\mathrm{S}$ & $\mathrm{C} 3$ & $\mathrm{H} 25$ & -180 & 15.167 & 2 \\
\hline $\mathrm{H} 25$ & $\mathrm{C} 3$ & $\mathrm{CB}$ & $\overline{C B}$ & -180 & 15.167 & 2 \\
\hline $\mathrm{S}$ & $\mathrm{CA}$ & $\mathrm{CB}$ & $\mathrm{H}$ & -180 & 15.167 & 2 \\
\hline $\mathrm{CA}$ & $\mathrm{CA}$ & $S$ & $\mathrm{CA}$ & -180 & 15.167 & 2 \\
\hline $\mathrm{CB}$ & CB & CA & $\mathrm{CA}$ & -180 & 15.167 & 2 \\
\hline C3 & $\mathrm{S}$ & $\overline{C A}$ & $\overline{\mathrm{CA}}$ & -180 & 15.167 & 2 \\
\hline CA & CA & $\mathrm{CB}$ & $\mathrm{H}$ & -180 & 15.167 & 2 \\
\hline$\overline{C A}$ & $\overline{\mathrm{CA}}$ & $\overline{C B}$ & $\mathrm{C} 10$ & -180 & 15.167 & 2 \\
\hline C3 & $\mathrm{CB}$ & CB & $\mathrm{C} 10$ & -180 & 15.167 & 2 \\
\hline $\bar{S}$ & CA & $\mathrm{CB}$ & $\mathrm{C} 10$ & -180 & 15.167 & 2 \\
\hline $\mathrm{H}$ & $\mathrm{CB}$ & $\mathrm{CB}$ & C10 & -180 & 15.167 & 2 \\
\hline $\mathrm{CA}$ & $\mathrm{CB}$ & $\mathrm{CB}$ & $\mathrm{C} 10$ & -180 & 15.167 & 2 \\
\hline$S$ & $\mathrm{C} 3$ & $\mathrm{CB}$ & C10 & -180 & 15.167 & 2 \\
\hline $\mathrm{H} 25$ & $\mathrm{C} 3$ & $\mathrm{CB}$ & C10 & -180 & 15.167 & 2 \\
\hline \multicolumn{7}{|c|}{ improper parameters } \\
\hline \multicolumn{4}{|c|}{ atom type } & $\phi_{n}\left({ }^{\circ}\right)$ & $k_{n}(\mathrm{~kJ} / \mathrm{mol})$ & $n$ \\
\hline C3 & $\mathrm{CB}$ & $\mathrm{S}$ & $\mathrm{H} 25$ & $\begin{array}{l}-180 \\
\end{array}$ & 4.6024 & 2 \\
\hline $\mathrm{CB}$ & $\overline{C B}$ & $\mathrm{CA}$ & $\mathrm{H}$ & -180 & 4.6024 & 2 \\
\hline$\overline{\mathrm{CA}}$ & $\overline{C B}$ & $\mathrm{~S}$ & $\overline{\mathrm{CA}}$ & -180 & 4.6024 & 2 \\
\hline$\overline{C B}$ & $\mathrm{CB}$ & $\mathrm{C} 3$ & $\mathrm{H}$ & -180 & 4.6024 & 2 \\
\hline $\mathrm{CB}$ & $\mathrm{CB}$ & $\mathrm{C} 3$ & $\mathrm{C} 10$ & -180 & 4.6024 & 2 \\
\hline $\mathrm{CB}$ & $\mathrm{CB}$ & $\mathrm{CA}$ & $\mathrm{C} 10$ & -180 & 4.6024 & 2 \\
\hline
\end{tabular}




\section{S1.4 FF-4 by Alberga et al. ${ }^{6}$}

The functional form of FF-4 is

$$
\begin{aligned}
& U=\sum_{\text {bonds }} \frac{k_{r}}{2}\left(r-r_{0}\right)^{2}+\sum_{\text {angles }} \frac{k_{\theta}}{2}\left(\theta-\theta_{0}\right)^{2}+\sum_{\text {dihedrals }} \sum_{n=1}^{m} k_{n}\left(1+\cos \left(n \phi-\phi_{n}\right)\right) \\
& +\sum_{\text {impropers }} \frac{k_{\xi}}{2}\left(\xi-\xi_{0}\right)^{2}+ \\
& \sum_{\text {nonbonded }}\left\{4 \varepsilon_{i j}\left[\left(\frac{\sigma_{i j}}{r_{i j}}\right)^{12}-\left(\frac{\sigma_{i j}}{r_{i j}}\right)^{6}\right]+\frac{C q_{i} q_{j}}{\varepsilon_{r} r_{i j}}\right\}
\end{aligned}
$$

The coding of atom types for RR-P3HT and the values of the force field parameters for the non-bonded and bonded interactions in FF-4 are presented in Table S1.4. 
Table S1.4. Potential form and parameters of FF-4.

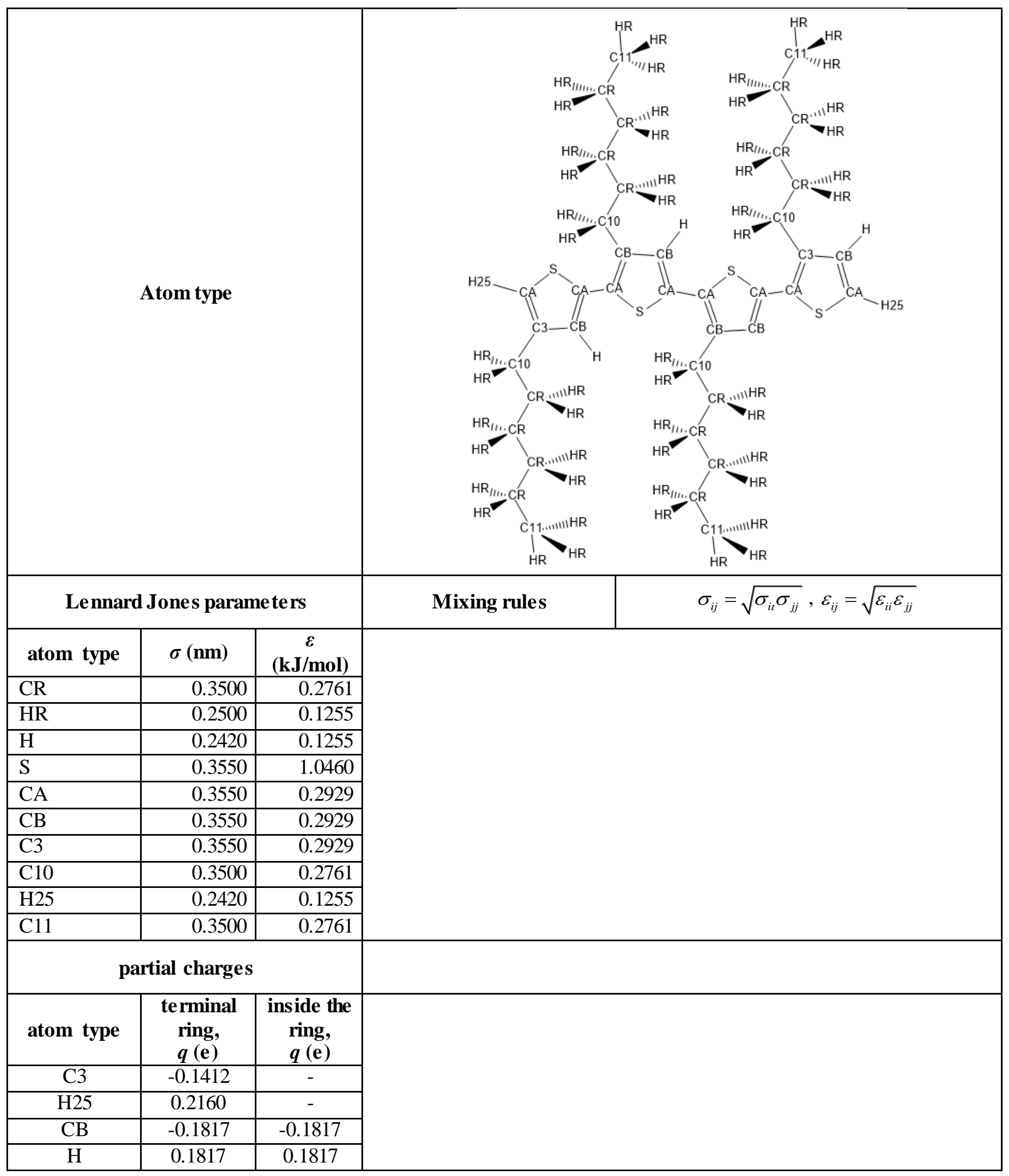




\begin{tabular}{|c|c|c|c|c|c|}
\hline $\mathrm{CA}$ & 0.0748 & 0.0748 & & & \\
\hline $\mathrm{S}$ & -0.1496 & -0.1496 & & & \\
\hline $\mathrm{C} 10$ & 0.0617 & 0.0617 & & & \\
\hline HR & 0.0600 & 0.0600 & & & \\
\hline CR & -0.1200 & -0.1200 & & & \\
\hline C11 & -0.1800 & -0.1800 & & & \\
\hline \multicolumn{4}{|c|}{ bond length streching parame ters } & & \\
\hline \multicolumn{2}{|c|}{ atom types } & $r_{0}(\mathrm{~nm})$ & $k_{r}(\mathrm{~kJ} / \mathbf{m o l})$ & & \\
\hline CR & $C R$ & 01520 & 2242624 & & \\
\hline $\mathrm{CR}$ & HR & 0.1090 & $\frac{22+\angle 02.4}{284512.0}$ & & \\
\hline $\mathrm{CB}$ & $\mathrm{H}$ & 0.1080 & 310143.2 & & \\
\hline CB & C10 & 0.1510 & 265265.6 & & \\
\hline $\mathrm{CA}$ & $\mathrm{CB}$ & 0.1364 & 430341.1 & & \\
\hline $\mathrm{S}$ & CA & 0.1732 & 243718.0 & & \\
\hline $\mathrm{CB}$ & $\mathrm{CB}$ & 0.1428 & 379154.1 & & \\
\hline $\mathrm{CA}$ & CA & 0.1430 & 328268.3 & & \\
\hline CB & $\mathrm{C} 3$ & 0.1364 & 430341.1 & & \\
\hline CR & $\overline{\mathrm{C} 10}$ & 0.1529 & 224262.4 & & \\
\hline C3 & $\mathrm{H} 25$ & 0.1080 & 310143.2 & & \\
\hline HR & C10 & 0.1090 & 284512.0 & & \\
\hline C3 & $\mathrm{S}$ & 0.1732 & 243718.0 & & \\
\hline C11 & CR & 0.1529 & 224262.4 & & \\
\hline C11 & $\mathrm{HR}$ & 0.1090 & 284512.0 & & \\
\hline \multicolumn{5}{|c|}{ bond streching parameters } & \\
\hline \multicolumn{3}{|c|}{ atom type } & $\theta_{0}\left({ }^{\circ}\right)$ & $k_{\theta}\left(\mathrm{kJ} / \mathrm{mol} \mathrm{rad}^{2}\right)$ & \\
\hline $\mathrm{CR}$ & $\mathrm{CR}$ & CR & 112.70 & 488.27 & \\
\hline CR & $\overline{\mathrm{CR}}$ & $\overline{\mathrm{HR}}$ & 110.70 & 313.80 & \\
\hline HR & $\overline{C R}$ & HR & 107.80 & 276.14 & \\
\hline CR & C10 & $\mathrm{CB}$ & 114.00 & 527.18 & \\
\hline $\mathrm{HR}$ & $\mathrm{C} 10$ & $\mathrm{CB}$ & 109.50 & 251.04 & \\
\hline $\mathrm{C} 10$ & $\mathrm{CB}$ & $\mathrm{CB}$ & 123.70 & 585.76 & \\
\hline $\mathrm{C} 10$ & $\mathrm{CB}$ & CA & 122.98 & 585.76 & \\
\hline $\mathrm{CB}$ & $\mathrm{CB}$ & CA & 113.32 & 331.22 & \\
\hline $\mathrm{H}$ & $\mathrm{CB}$ & $\mathrm{CB}$ & 123.70 & 295.08 & \\
\hline $\mathrm{H}$ & $\mathrm{CB}$ & $\overline{\mathrm{CA}}$ & 122.98 & 295.08 & \\
\hline CA & $\mathrm{S}$ & CA & 92.77 & 722.66 & \\
\hline $\mathrm{CA}$ & $\mathrm{CA}$ & $\mathrm{CB}$ & 130.14 & 457.68 & \\
\hline $\bar{S}$ & $\mathrm{CA}$ & $\mathrm{CA}$ & 119.57 & 349.28 & \\
\hline$S$ & CA & $\mathrm{CB}$ & 110.29 & 722.66 & \\
\hline $\mathrm{H} 25$ & $\mathrm{C} 3$ & $\mathrm{~S}$ & 124.61 & 240.89 & \\
\hline $\mathrm{H} 25$ & $\mathrm{C} 3$ & $\mathrm{CB}$ & 125.10 & 295.08 & \\
\hline C3 & $\mathrm{CB}$ & $\mathrm{CB}$ & 113.32 & 331.22 & \\
\hline
\end{tabular}




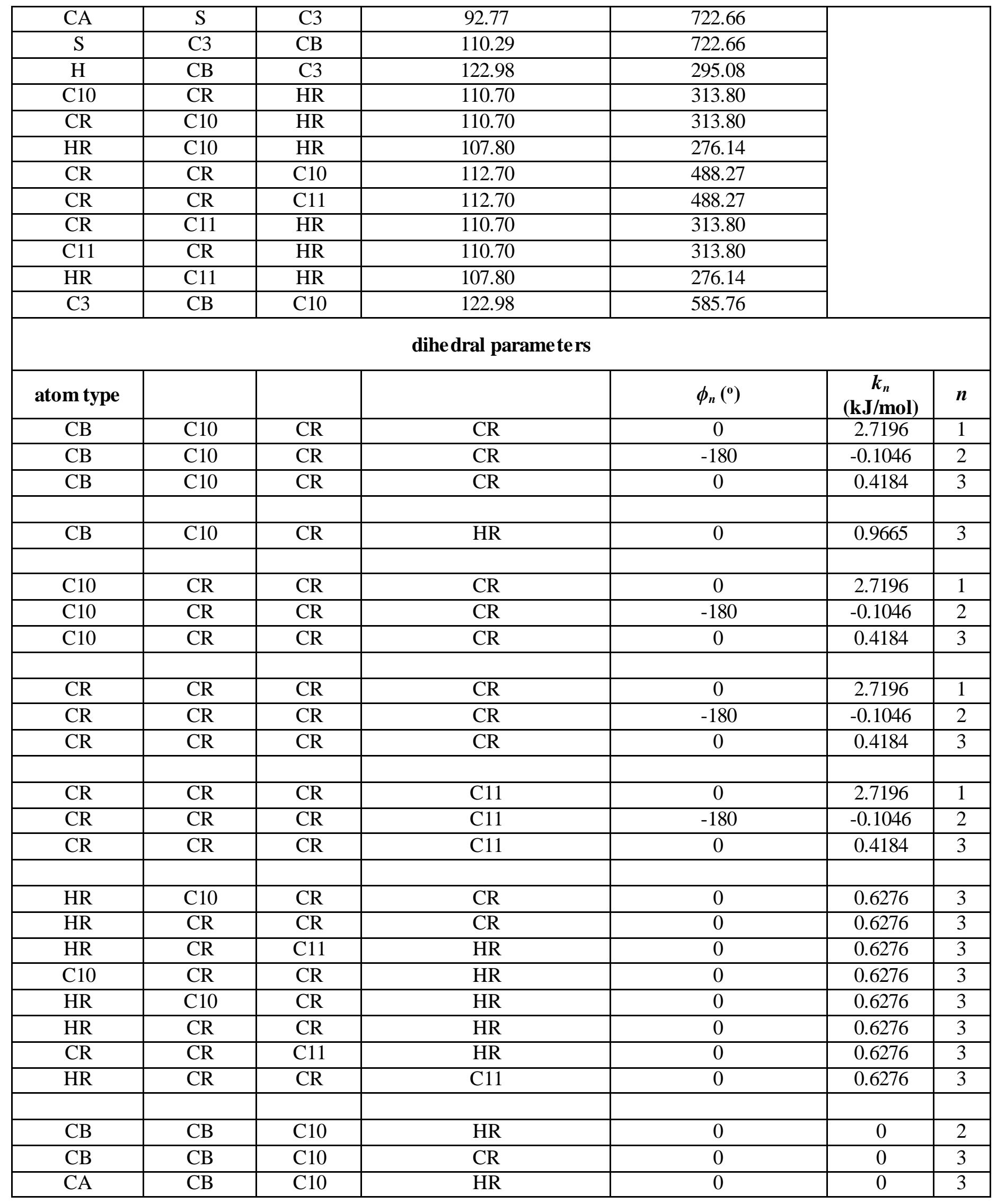




\begin{tabular}{|c|c|c|c|c|c|c|}
\hline C3 & $\mathrm{CB}$ & $\mathrm{C} 10$ & $\overline{\mathrm{HR}}$ & 0 & 0 & 3 \\
\hline CA & $\mathrm{CB}$ & $\mathrm{C} 10$ & CR & 0 & 0 & 3 \\
\hline C3 & $\mathrm{CB}$ & $\mathrm{C} 10$ & CR & 0 & 0 & 3 \\
\hline CB & CA & CA & $\mathrm{S}$ & 0 & 0 & 3 \\
\hline CB & CA & $\mathrm{CA}$ & $\mathrm{CB}$ & 0 & 0 & 3 \\
\hline $\mathrm{S}$ & $\mathrm{CA}$ & CA & $\mathrm{S}$ & 0 & -0.32761 & 1 \\
\hline $\mathrm{S}$ & $\mathrm{CA}$ & $\mathrm{CA}$ & $\mathrm{S}$ & -180 & 11.33529 & 2 \\
\hline $\mathrm{S}$ & CA & CA & $\mathrm{S}$ & 0 & 0.2979 & 3 \\
\hline C3 & $\mathrm{S}$ & CA & $\mathrm{CB}$ & -180 & 25.71696 & 2 \\
\hline C3 & $\mathrm{CB}$ & $\mathrm{CB}$ & $\overline{C A}$ & 0 & -2.07108 & 1 \\
\hline C3 & $\mathrm{CB}$ & $\mathrm{CB}$ & $\mathrm{CA}$ & -180 & 6.60653 & 2 \\
\hline & & & & & & \\
\hline CB & $\mathrm{CB}$ & CA & $\mathrm{S}$ & -180 & 38.77522 & 2 \\
\hline CB & $\mathrm{CB}$ & CA & $\mathrm{S}$ & 0 & 12.552 & 3 \\
\hline $\mathrm{CB}$ & C3 & $\mathrm{S}$ & $\mathrm{CA}$ & -180 & 27.64787 & 2 \\
\hline $\mathrm{CB}$ & $\mathrm{CB}$ & $\bar{C} 3$ & $\mathrm{~S}$ & -180 & 43.56799 & 2 \\
\hline CB & CB & C3 & $\mathrm{S}$ & 0 & 12.552 & 3 \\
\hline $\mathrm{CA}$ & $\mathrm{CB}$ & $\mathrm{CB}$ & $\mathrm{CA}$ & 0 & -2.07108 & 1 \\
\hline $\mathrm{CA}$ & $\mathrm{CB}$ & $\mathrm{CB}$ & $\mathrm{CA}$ & -180 & 6.90988 & 2 \\
\hline $\mathrm{CA}$ & $\mathrm{S}$ & $\mathrm{CA}$ & $\mathrm{CB}$ & -180 & 28.0056 & 2 \\
\hline $\mathrm{CA}$ & $\mathrm{CB}$ & $\mathrm{CB}$ & $\mathrm{H}$ & 0 & 0.523 & 1 \\
\hline $\mathrm{CA}$ & $\mathrm{CB}$ & $\mathrm{CB}$ & $\mathrm{H}$ & -180 & 7.9747 & 2 \\
\hline CA & CB & $\mathrm{CB}$ & $\mathrm{H}$ & 0 & -1.1506 & 3 \\
\hline C3 & $\mathrm{CB}$ & $\mathrm{CB}$ & $\mathrm{H}$ & 0 & 0.523 & 1 \\
\hline C3 & $\mathrm{CB}$ & $\mathrm{CB}$ & $\mathrm{H}$ & -180 & 7.62011 & 2 \\
\hline C3 & $\mathrm{CB}$ & $\mathrm{CB}$ & $\mathrm{H}$ & 0 & -1.1506 & 3 \\
\hline$S$ & $\mathrm{C} 3$ & $\mathrm{CB}$ & $\mathrm{H}$ & 0 & -8.87426 & 1 \\
\hline $\mathrm{S}$ & $\mathrm{C} 3$ & $\mathrm{CB}$ & $\mathrm{H}$ & $\begin{array}{l}-180 \\
\end{array}$ & 13.64402 & 2 \\
\hline $\mathrm{H} 25$ & $\mathrm{C} 3$ & $\mathrm{CB}$ & $\mathrm{H}$ & -180 & 21.20452 & 2 \\
\hline $\mathrm{CA}$ & $\mathrm{S}$ & C3 & $\mathrm{H} 25$ & -180 & 1.94974 & 2 \\
\hline $\mathrm{H} 25$ & $\mathrm{C} 3$ & $\mathrm{CB}$ & $\mathrm{CB}$ & 0 & 0.523 & 1 \\
\hline $\mathrm{H} 25$ & $\mathrm{C} 3$ & $\mathrm{CB}$ & $\mathrm{CB}$ & -180 & 16.59374 & 2 \\
\hline $\mathrm{H} 25$ & $\mathrm{C} 3$ & $\mathrm{CB}$ & $\mathrm{CB}$ & 0 & -1.1506 & 3 \\
\hline
\end{tabular}




\begin{tabular}{|c|c|c|c|c|c|c|}
\hline $\mathrm{S}$ & $\mathrm{CA}$ & $\mathrm{CB}$ & $\mathrm{H}$ & 0 & -8.87426 & 1 \\
\hline$S$ & $\mathrm{CA}$ & $\mathrm{CB}$ & $\mathrm{H}$ & -180 & 12.14197 & 2 \\
\hline $\mathrm{CA}$ & $\mathrm{CA}$ & $\mathrm{S}$ & $\mathrm{CA}$ & -180 & 159829 & 2 \\
\hline $\mathrm{CA}$ & $\mathrm{CA}$ & $\mathrm{S}$ & $\mathrm{CA}$ & 0 & 0.8368 & 3 \\
\hline CB & $\mathrm{CB}$ & $\mathrm{CA}$ & $\mathrm{CA}$ & 0 & -1.40164 & 1 \\
\hline $\mathrm{CB}$ & $\mathrm{CB}$ & $\mathrm{CA}$ & $\mathrm{CA}$ & -180 & 16.40965 & 2 \\
\hline $\mathrm{C} 3$ & $\mathrm{~S}$ & $\mathrm{CA}$ & $\mathrm{CA}$ & -180 & 1.60247 & 2 \\
\hline $\mathrm{C} 3$ & $\mathrm{~S}$ & $\mathrm{CA}$ & $\mathrm{CA}$ & 0 & 0.8368 & 3 \\
\hline $\mathrm{CA}$ & $\mathrm{CA}$ & $\mathrm{CB}$ & $\bar{H}$ & 0 & 0.523 & $\overline{1}$ \\
\hline $\mathrm{CA}$ & $\mathrm{CA}$ & CB & $\mathrm{H}$ & -180 & 14.76743 & 2 \\
\hline $\mathrm{CA}$ & $\mathrm{CA}$ & $\mathrm{CB}$ & $\bar{H}$ & 0 & -1.1506 & 3 \\
\hline $\mathrm{CA}$ & $\mathrm{CA}$ & $\mathrm{CB}$ & $\mathrm{C} 10$ & 0 & 0.523 & 1 \\
\hline $\mathrm{CA}$ & $\mathrm{CA}$ & $\mathrm{CB}$ & C10 & -180 & 14.76743 & 2 \\
\hline $\mathrm{CA}$ & $\mathrm{CA}$ & CB & C10 & 0 & -1.1506 & 3 \\
\hline $\mathrm{C} 3$ & $\mathrm{CB}$ & $\mathrm{CB}$ & $\mathrm{C} 10$ & 0 & 0.523 & 1 \\
\hline $\mathrm{C} 3$ & $\mathrm{CB}$ & $\mathrm{CB}$ & C10 & -180 & 7.62116 & 2 \\
\hline C3 & CB & CB & C10 & 0 & -1.1506 & 3 \\
\hline $\bar{S}$ & $\overline{\mathrm{CA}}$ & $\mathrm{CB}$ & $\mathrm{C} 10$ & 0 & -8.87426 & 1 \\
\hline $\mathrm{S}$ & $\mathrm{CA}$ & CB & C10 & -180 & 12.14197 & 2 \\
\hline $\mathrm{H}$ & $\mathrm{CB}$ & $\mathrm{CB}$ & $\mathrm{C} 10$ & -180 & 10.18804 & 2 \\
\hline $\mathrm{CA}$ & $\mathrm{CB}$ & $\mathrm{CB}$ & $\mathrm{C} 10$ & 0 & 0.523 & 1 \\
\hline $\mathrm{CA}$ & $\mathrm{CB}$ & CB & C10 & -180 & 7.9747 & 2 \\
\hline $\mathrm{CA}$ & $\mathrm{CB}$ & CB & C10 & 0 & -1.1506 & 3 \\
\hline $\mathrm{S}$ & C3 & $\mathrm{CB}$ & C10 & 0 & -8.87426 & 1 \\
\hline $\bar{S}$ & C3 & $\mathrm{CB}$ & C10 & -180 & 13.64402 & 2 \\
\hline $\mathrm{H} 25$ & C3 & CB & $\mathrm{C} 10$ & -180 & 21.20451 & 2 \\
\hline \multicolumn{7}{|c|}{ improper parameters } \\
\hline atom type & & & & $\xi_{0}\left({ }^{0}\right)$ & $\begin{array}{c}\boldsymbol{k}_{\xi} \\
(\mathrm{kJ} / \mathrm{mol})\end{array}$ & \\
\hline $\mathrm{C} 3$ & $\mathrm{CB}$ & $\mathrm{S}$ & $\mathrm{H} 25$ & 0 & 167.36 & \\
\hline$\overline{C B}$ & $\mathrm{CB}$ & $\overline{\mathrm{CA}}$ & $\overline{\mathrm{H}}$ & $\overline{0}$ & 167.36 & \\
\hline$\overline{\mathrm{CA}}$ & $\mathrm{S}$ & $\mathrm{CB}$ & $\mathrm{CA}$ & $\overline{0}$ & 167.36 & \\
\hline CB & $\mathrm{CB}$ & C3 & $\mathrm{H}$ & 0 & 167.36 & \\
\hline
\end{tabular}




\section{S1.5 FF-5 by Moreno et al. ${ }^{7}$}

The functional form of FF-5 is

$$
\begin{aligned}
& U=\sum_{\text {bonds }} \frac{k_{l}}{2}\left(r-r_{0}\right)^{2}+\sum_{\text {angles }} \frac{k_{\theta}}{2}\left(\theta-\theta_{0}\right)^{2}+\sum_{\text {dihedrals }} \sum_{n=1}^{m} k_{n}\left(1+\cos \left(n \phi-\phi_{n}\right)\right) \\
& +\sum_{\text {impropers }} \sum_{n=1}^{m} k_{n}\left(1+\cos \left(n \phi-\phi_{n}\right)\right)+ \\
& \sum_{\text {nonbonded }}\left\{4 \varepsilon_{i j}\left[\left(\frac{\sigma_{i j}}{r_{i j}}\right)^{12}-\left(\frac{\sigma_{i j}}{r_{i j}}\right)^{6}\right]+\frac{C q_{i} q_{j}}{\varepsilon_{r} r_{i j}}\right\}
\end{aligned}
$$

The coding of atom types for RR-P3HT and the values of the force field parameters for the non-bonded and bonded interactions in FF-5 are presented in Table S1.5. 
Table S1.5. Potential form and parameters of FF-5.

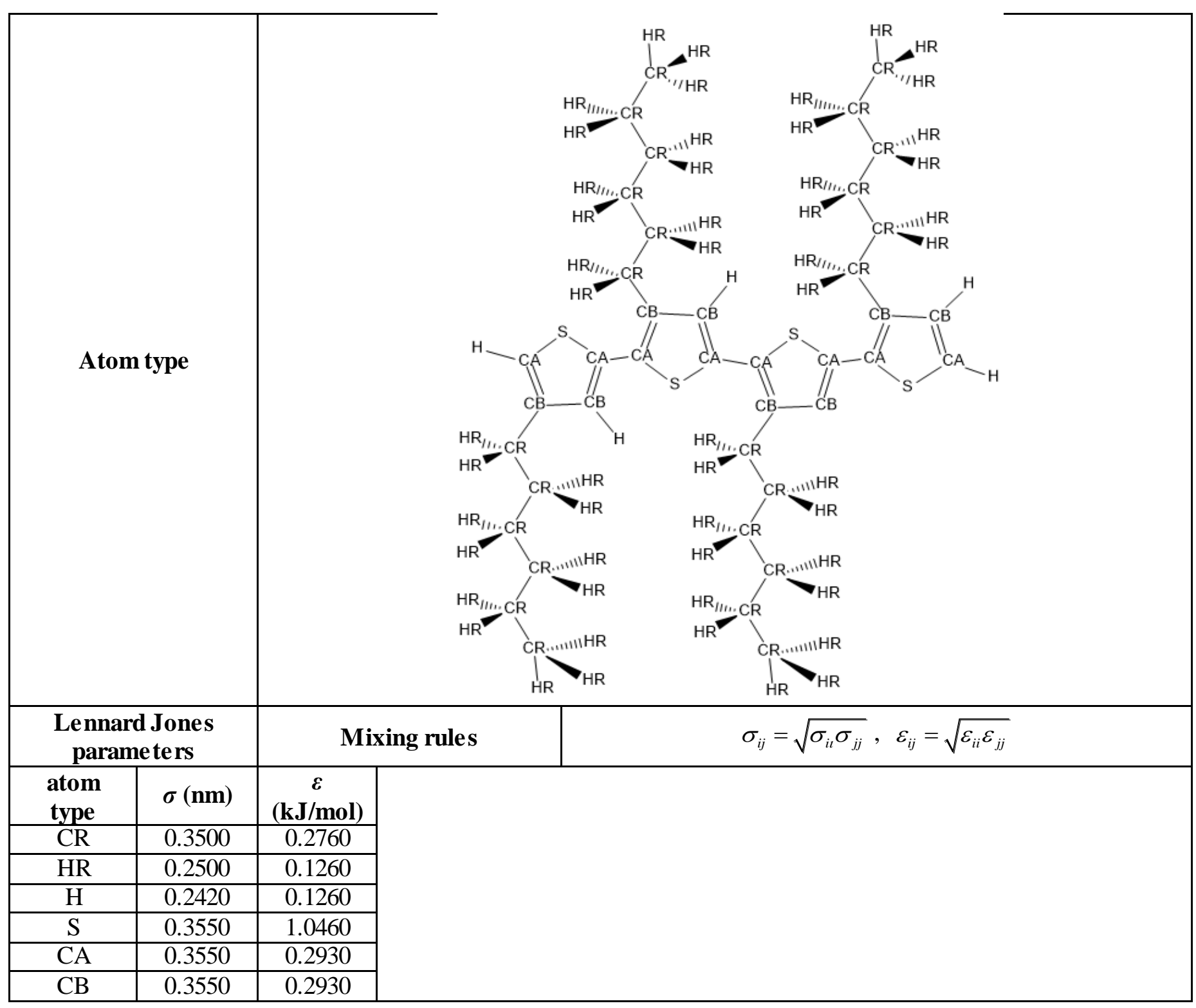




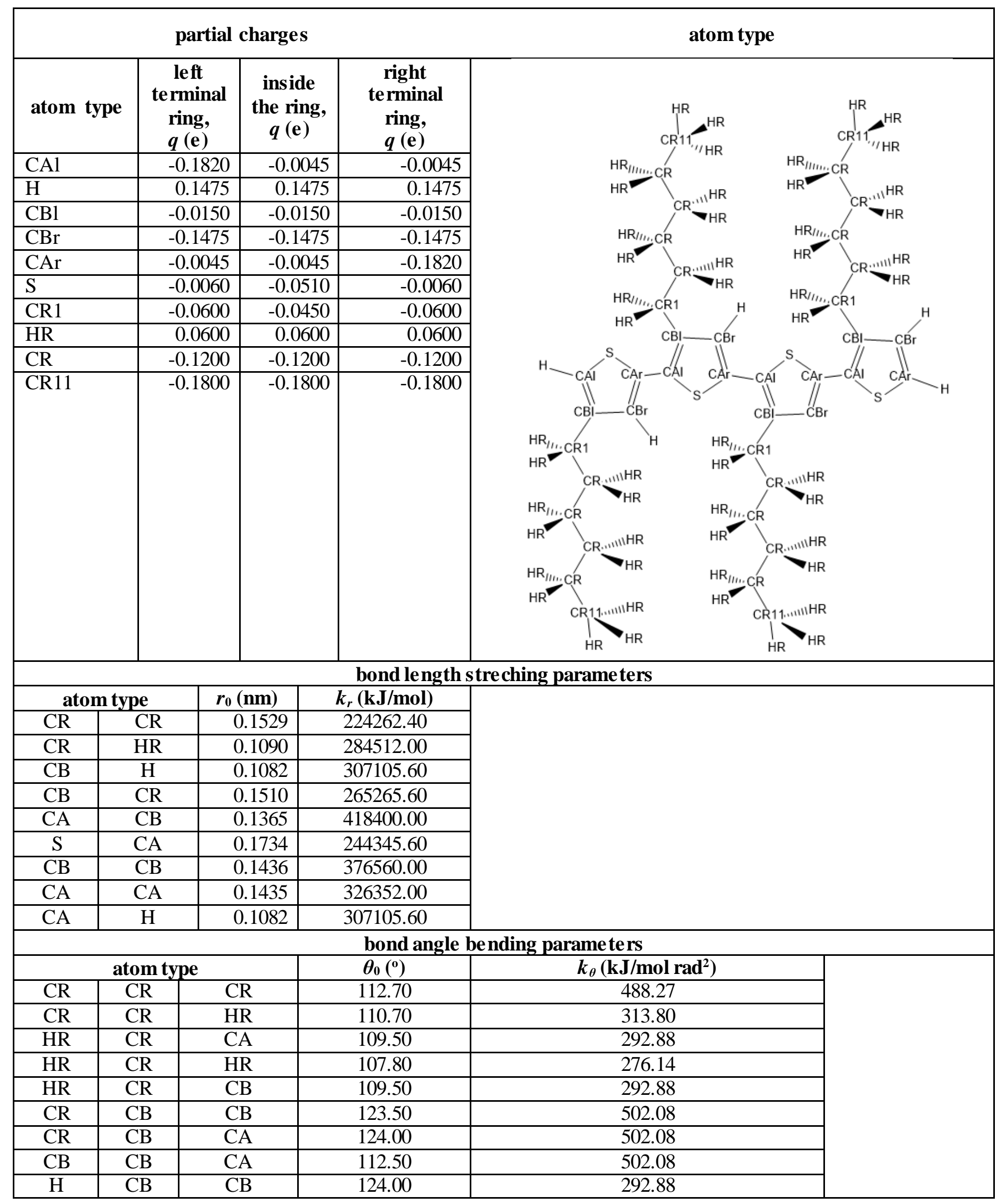




\begin{tabular}{|c|c|c|c|c|}
\hline $\mathrm{H}$ & $\mathrm{CB}$ & $\mathrm{CA}$ & 123.50 & 292.88 \\
\hline $\mathrm{CA}$ & $\mathrm{S}$ & $\mathrm{CA}$ & 91.00 & 502.08 \\
\hline $\mathrm{CA}$ & $\mathrm{CA}$ & $\mathrm{CB}$ & 129.00 & 502.08 \\
\hline $\mathrm{S}$ & $\mathrm{CA}$ & $\mathrm{CA}$ & 119.00 & 502.08 \\
\hline $\mathrm{S}$ & $\mathrm{CA}$ & $\mathrm{CB}$ & 112.00 & 502.08 \\
\hline $\mathrm{H}$ & $\mathrm{CA}$ & $\mathrm{S}$ & 119.00 & 502.08 \\
\hline $\mathrm{H}$ & $\mathrm{CA}$ & $\mathrm{CB}$ & 129.00 & 502.08 \\
\hline
\end{tabular}

dihe dral parameters

\begin{tabular}{|c|c|c|c|c|c|c|}
\hline \multicolumn{4}{|c|}{ atom type } & $\phi_{n}\left({ }^{\circ}\right)$ & $k_{n}(\mathrm{~kJ} / \mathrm{mol})$ & $n$ \\
\hline $\mathrm{CR}$ & $\mathrm{CR}$ & $\mathrm{CR}$ & CR & 0 & 2.7196 & 1 \\
\hline $\mathrm{CR}$ & $\mathrm{CR}$ & $\mathrm{CR}$ & CR & 180 & -0.1046 & 2 \\
\hline $\mathrm{CR}$ & $\mathrm{CR}$ & $\mathrm{CR}$ & CR & 0 & 0.4184 & 3 \\
\hline $\mathrm{CR}$ & CR & $\mathrm{CR}$ & HR & 0 & 0.6276 & 3 \\
\hline HR & $\mathrm{CR}$ & $\mathrm{CR}$ & HR & 0 & 0.6276 & 3 \\
\hline $\mathrm{HR}$ & $\mathrm{CR}$ & $\mathrm{CR}$ & $\mathrm{CB}$ & 0 & 0.9665 & 3 \\
\hline $\mathrm{CR}$ & $\mathrm{CR}$ & $\mathrm{CR}$ & $\mathrm{CB}$ & 0 & 2.7196 & 1 \\
\hline $\mathrm{CR}$ & $\mathrm{CR}$ & $\mathrm{CR}$ & $\mathrm{CB}$ & 180 & -0.1046 & 2 \\
\hline $\mathrm{CR}$ & $\mathrm{CR}$ & $\mathrm{CR}$ & $\mathrm{CB}$ & 0 & 0.4184 & 3 \\
\hline HR & $\mathrm{CR}$ & $\mathrm{CB}$ & $\mathrm{CB}$ & 0 & 0 & 1 \\
\hline HR & $\mathrm{CR}$ & $\mathrm{CB}$ & $\mathrm{CA}$ & 0 & 0 & 1 \\
\hline $\mathrm{CR}$ & $\mathrm{CR}$ & CB & $\mathrm{CB}$ & 0 & 0 & 1 \\
\hline CA & CB & CR & CR & 0 & -0.6109 & 1 \\
\hline $\mathrm{CA}$ & $\mathrm{CB}$ & $\mathrm{CR}$ & CR & 0 & 0.0084 & 2 \\
\hline $\mathrm{CA}$ & $\mathrm{CB}$ & $\mathrm{CR}$ & CR & 0 & -1.9121 & 3 \\
\hline $\mathrm{CA}$ & $\mathrm{CB}$ & $\mathrm{CR}$ & $\mathrm{CR}$ & 0 & -0.2322 & 4 \\
\hline $\mathrm{S}$ & $\mathrm{CA}$ & $\mathrm{CA}$ & $\mathrm{CB}$ & 0 & 0 & 1 \\
\hline $\mathrm{CB}$ & $\mathrm{CA}$ & $\mathrm{CA}$ & $\mathrm{CB}$ & 0 & 0 & 1 \\
\hline $\bar{S}$ & CA & CA & $\mathrm{S}$ & 0 & 1.2468 & 1 \\
\hline $\mathrm{S}$ & $\mathrm{CA}$ & $\mathrm{CA}$ & $\mathrm{S}$ & 0 & -8.4496 & 2 \\
\hline$S$ & $\mathrm{CA}$ & $\mathrm{CA}$ & $\mathrm{S}$ & 0 & 0.5272 & 3 \\
\hline $\mathrm{S}$ & $\mathrm{CA}$ & $\mathrm{CA}$ & $\mathrm{S}$ & 0 & 1.8012 & 4 \\
\hline$S$ & $\mathrm{CA}$ & $\mathrm{CA}$ & $S$ & 0 & 0.2678 & 5 \\
\hline $\mathrm{CA}$ & CB & CB & $\mathrm{CA}$ & 180 & 15.167 & 2 \\
\hline $\mathrm{H}$ & CB & CB & $\mathrm{CA}$ & 180 & 15.167 & 2 \\
\hline $\mathrm{H}$ & CB & CB & CR & 180 & 15.167 & 2 \\
\hline CA & CB & CB & CR & 180 & 15.167 & 2 \\
\hline $\mathrm{CB}$ & $\mathrm{CB}$ & $\mathrm{CA}$ & $\mathrm{S}$ & 180 & 15.167 & 2 \\
\hline CB & CB & $\mathrm{CA}$ & $\mathrm{CA}$ & 180 & 15.167 & 2 \\
\hline $\mathrm{CR}$ & CB & $\mathrm{CA}$ & $\mathrm{S}$ & 180 & 15.167 & 2 \\
\hline CR & CB & $\mathrm{CA}$ & $\mathrm{CA}$ & 180 & 15.167 & 2 \\
\hline $\mathrm{H}$ & CB & $\mathrm{CA}$ & $\mathrm{S}$ & 180 & 15.167 & 2 \\
\hline $\mathrm{H}$ & CB & $\mathrm{CA}$ & $\mathrm{CA}$ & 180 & 15.167 & 2 \\
\hline $\mathrm{CB}$ & $\mathrm{CB}$ & $\mathrm{CA}$ & $\mathrm{H}$ & 180 & 15.167 & 2 \\
\hline
\end{tabular}




\begin{tabular}{|c|c|c|c|c|c|c|c|}
\hline $\mathrm{CR}$ & $\mathrm{CB}$ & $\mathrm{CA}$ & $\mathrm{H}$ & 180 & 15.167 & \multicolumn{2}{|l|}{2} \\
\hline $\mathrm{H}$ & $\mathrm{CB}$ & $\mathrm{CA}$ & $\mathrm{H}$ & 180 & 15.167 & \multicolumn{2}{|l|}{2} \\
\hline $\mathrm{CA}$ & $\mathrm{S}$ & $\mathrm{CA}$ & $\overline{\mathrm{CA}}$ & 180 & 15.167 & \multicolumn{2}{|l|}{2} \\
\hline CA & $\mathrm{S}$ & $\mathrm{CA}$ & CB & 180 & 15.167 & \multicolumn{2}{|l|}{2} \\
\hline $\mathrm{CA}$ & $\mathrm{S}$ & $\mathrm{CA}$ & $\mathrm{H}$ & 180 & 15.167 & \multicolumn{2}{|l|}{2} \\
\hline \multicolumn{8}{|c|}{ improper parame te rs } \\
\hline \multicolumn{4}{|c|}{ atom type } & \multicolumn{2}{|c|}{$\phi_{n}\left({ }^{0}\right)$} & $k_{n}(\mathrm{~kJ} / \mathrm{mol})$ & $N$ \\
\hline CA & S & CB & CA & \multicolumn{2}{|c|}{180} & 9.2048 & 2 \\
\hline $\mathrm{CA}$ & $\mathrm{CB}$ & $S$ & $\mathrm{H}$ & \multicolumn{2}{|c|}{180} & 9.2048 & 2 \\
\hline CB & $\mathrm{CB}$ & $\mathrm{CA}$ & $\mathrm{H}$ & \multicolumn{2}{|c|}{180} & 9.2048 & 2 \\
\hline $\mathrm{CB}$ & $\mathrm{CB}$ & $\mathrm{CA}$ & CR & \multicolumn{2}{|c|}{180} & 9.2048 & 2 \\
\hline
\end{tabular}




\section{S1.6 FF-6 by Poelking et al. ${ }^{2}$}

The functional form of FF- 6 is

$$
\begin{aligned}
& U=\sum_{\text {bonds }} \frac{k_{r}}{2}\left(r-r_{0}\right)^{2}+\sum_{\text {angles }} \frac{k_{\theta}}{2}\left(\theta-\theta_{0}\right)^{2}+\sum_{\text {dihedrals }} \sum_{n=0}^{5} C_{n}(\cos (\phi))^{n} \\
& +\sum_{\text {impropers }} \frac{k_{\xi}}{2}\left(\xi-\xi_{0}\right)^{2}+ \\
& \sum_{\text {nonbonded }}\left\{4 \varepsilon_{i j}\left[\left(\frac{\sigma_{i j}}{r_{i j}}\right)^{12}-\left(\frac{\sigma_{i j}}{r_{i j}}\right)^{6}\right]+\frac{C q_{i} q_{j}}{\varepsilon_{r} r_{i j}}\right\}
\end{aligned}
$$

The coding of atom types for RR-P3HT and the values of the force field parameters for the non-bonded and bonded interactions in FF-6 are presented in Table S1.6. 
Table S1.6. Potential form and parameters of FF-6.

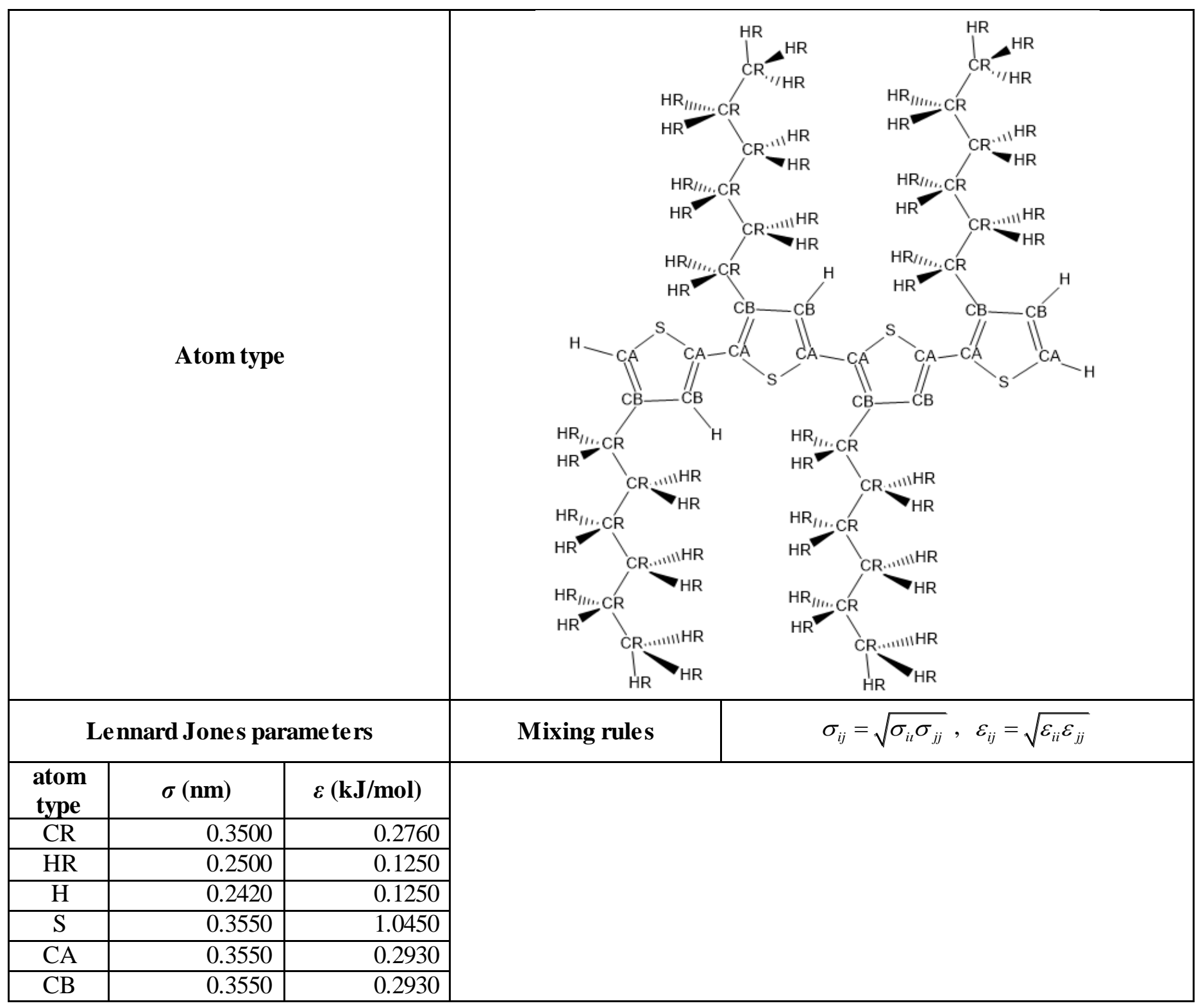




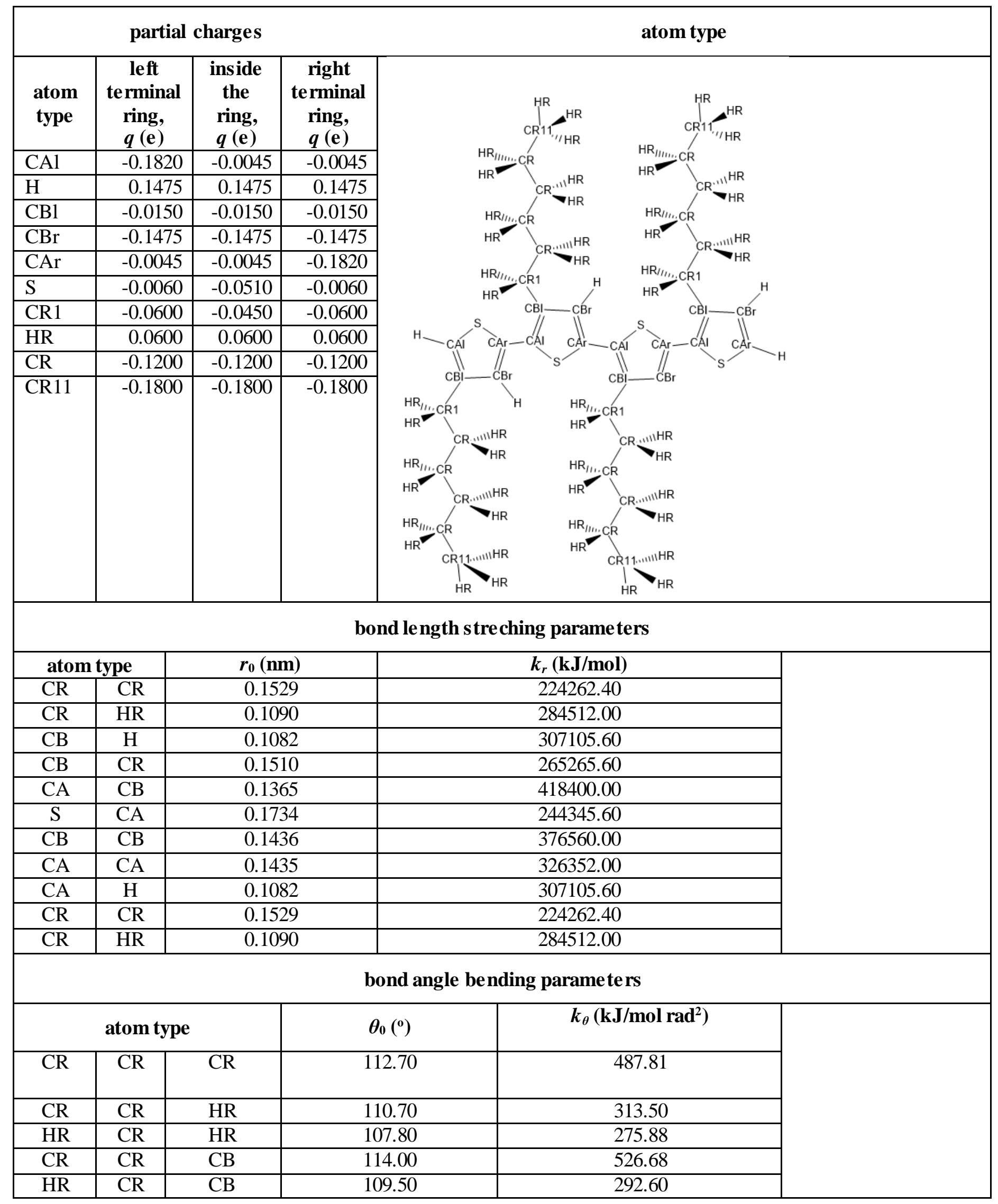




\begin{tabular}{|c|c|c|c|c|c|c|c|c|c|}
\hline CR & $\mathrm{CB}$ & \multicolumn{2}{|c|}{ CB } & \multicolumn{2}{|l|}{122.71} & \multicolumn{2}{|c|}{382.60} & & \\
\hline $\mathrm{CR}$ & $\mathrm{CB}$ & \multicolumn{2}{|c|}{$\mathrm{CA}$} & \multicolumn{2}{|l|}{123.62} & \multicolumn{2}{|c|}{382.60} & & \\
\hline$\overline{\mathrm{CB}}$ & $\overline{C B}$ & \multicolumn{2}{|c|}{$\overline{\mathrm{CA}}$} & \multicolumn{2}{|l|}{113.67} & \multicolumn{2}{|c|}{501.60} & & \\
\hline $\mathrm{H}$ & $\mathrm{CB}$ & \multicolumn{2}{|c|}{$\mathrm{CB}$} & \multicolumn{2}{|l|}{124.00} & \multicolumn{2}{|c|}{292.60} & & \\
\hline $\mathrm{H}$ & $\mathrm{CB}$ & \multicolumn{2}{|c|}{$\mathrm{CA}$} & \multicolumn{2}{|l|}{123.50} & \multicolumn{2}{|c|}{292.60} & & \\
\hline $\mathrm{CA}$ & $S$ & \multicolumn{2}{|c|}{ CA } & \multicolumn{2}{|l|}{92.50} & \multicolumn{2}{|c|}{501.60} & & \\
\hline $\mathrm{CA}$ & $\mathrm{CA}$ & \multicolumn{2}{|c|}{$\mathrm{CB}$} & \multicolumn{2}{|l|}{128.49} & \multicolumn{2}{|c|}{480.35} & & \\
\hline $\mathrm{S}$ & $\mathrm{CA}$ & \multicolumn{2}{|c|}{$\mathrm{CA}$} & \multicolumn{2}{|l|}{121.52} & \multicolumn{2}{|c|}{480.35} & & \\
\hline$S$ & $\mathrm{CA}$ & \multicolumn{2}{|c|}{$\mathrm{CB}$} & 110.08 & & & 01.60 & & \\
\hline $\mathrm{H}$ & $\overline{\mathrm{CA}}$ & & & 119.00 & & & 01.60 & & \\
\hline $\mathrm{H}$ & $\mathrm{CA}$ & & & 129.00 & & & $\overline{11.60}$ & & \\
\hline & & & & & hedral par & meters & & & \\
\hline & ato & type & & $\begin{array}{c}\mathrm{C}_{\mathbf{0}} \\
(\mathrm{kJ} / \mathrm{mol})\end{array}$ & $\begin{array}{c}\mathrm{C}_{1} \\
(\mathrm{~kJ} / \mathrm{mol})\end{array}$ & $\begin{array}{c}\mathrm{C}_{2} \\
(\mathrm{~kJ} / \mathrm{mol})\end{array}$ & $\begin{array}{c}\mathrm{C}_{3} \\
(\mathrm{~kJ} / \mathrm{mol})\end{array}$ & $\begin{array}{c}\mathrm{C}_{4} \\
(\mathrm{~kJ} / \mathrm{mol})\end{array}$ & $\begin{array}{c}\mathrm{C}_{5} \\
(\mathrm{~kJ} / \mathrm{mol})\end{array}$ \\
\hline $\mathrm{CR}$ & CR & $\mathrm{CR}$ & CR & 2.9288 & -1.4644 & 0.2092 & -1.6736 & 0.0000 & 0.0000 \\
\hline$\overline{\mathrm{CR}}$ & $\overline{C R}$ & $\overline{C R}$ & \begin{tabular}{|l|}
$\mathrm{HR}$ \\
\end{tabular} & 0.6276 & 1.8828 & 0.0000 & -2.5104 & 0.0000 & 0.0000 \\
\hline HR & $\overline{\mathrm{CR}}$ & $\overline{C R}$ & HR & 0.6276 & 1.8828 & 0.0000 & -2.5104 & 0.0000 & 0.0000 \\
\hline HR & $\mathrm{CR}$ & CR & CB & 0.9660 & 2.8970 & 0.0000 & -3.8620 & 0.0000 & 0.0000 \\
\hline$\overline{\mathrm{CR}}$ & $\overline{\mathrm{CR}}$ & $\overline{C R}$ & $\mathrm{CB}$ & 2.9288 & -1.4644 & 0.2092 & -1.6736 & 0.0000 & 0.0000 \\
\hline HR & $\overline{\mathrm{CR}}$ & $\mathrm{CB}$ & $\mathrm{CB}$ & 0.0000 & 0.0000 & 0.0000 & 0.0000 & 0.0000 & 0.0000 \\
\hline HR & $\mathrm{CR}$ & $\mathrm{CB}$ & CA & 0.0000 & 0.0000 & 0.0000 & 0.0000 & 0.0000 & 0.0000 \\
\hline$\overline{\mathrm{CR}}$ & $\overline{C R}$ & $\mathrm{CB}$ & $\mathrm{CB}$ & 0.0000 & 0.0000 & 0.0000 & 0.0000 & 0.0000 & 0.0000 \\
\hline $\mathrm{CA}$ & $\mathrm{CB}$ & CR & CR & 0.5016 & -7.2488 & -3.9434 & 7.2249 & 0.1234 & 0.0000 \\
\hline$S$ & $\mathrm{CA}$ & $\mathrm{CA}$ & CB & 0.0000 & 0.0000 & 0.0000 & 0.0000 & 0.0000 & 0.0000 \\
\hline$\overline{\mathrm{CB}}$ & $\overline{\mathrm{CA}}$ & $\overline{\mathrm{CA}}$ & $\mathrm{CB}$ & 0.0000 & 0.0000 & 0.0000 & 0.0000 & 0.0000 & 0.0000 \\
\hline $\mathrm{S}$ & $\mathrm{CA}$ & $\mathrm{CA}$ & $\mathrm{S}$ & 21.7910 & -0.2076 & -14.2695 & 0.4739 & -6.6518 & 0.0000 \\
\hline$\overline{\mathrm{CA}}$ & $\mathrm{CB}$ & $\mathrm{CB}$ & $\mathrm{CA}$ & 30.3050 & 0.0000 & -30.3050 & 0.0000 & 0.0000 & 0.0000 \\
\hline $\mathrm{H}$ & $\mathrm{CB}$ & $\mathrm{CB}$ & CA & 30.3050 & 0.0000 & -30.3050 & 0.0000 & 0.0000 & 0.0000 \\
\hline $\mathrm{H}$ & $\mathrm{CB}$ & $\mathrm{CB}$ & CR & 30.3050 & 0.0000 & -30.3050 & 0.0000 & 0.0000 & 0.0000 \\
\hline $\mathrm{CA}$ & $\mathrm{CB}$ & CB & CR & 30.3050 & 0.0000 & -30.3050 & 0.0000 & 0.0000 & 0.0000 \\
\hline & & & & & proper pal & meters & & & \\
\hline & & om ty & & & ${ }_{0}\left({ }^{\circ}\right)$ & $\begin{array}{c}\boldsymbol{k}_{\xi} \\
(\mathrm{kJ} / \mathrm{mol})\end{array}$ & & & \\
\hline $\mathrm{CA}$ & $\mathrm{S}$ & $\mathrm{CA}$ & $\mathrm{CB}$ & & 0.00 & 160.00 & & & \\
\hline $\mathrm{CA}$ & CB & $\mathrm{CB}$ & $\mathrm{CA}$ & & 0.00 & 160.00 & & & \\
\hline $\mathrm{CB}$ & CB & $\mathrm{CA}$ & $\mathrm{S}$ & & 0.00 & 160.00 & & & \\
\hline $\mathrm{CB}$ & $\mathrm{CB}$ & $\mathrm{CA}$ & $\mathrm{H}$ & & 0.00 & 430.70 & & & \\
\hline$\overline{\mathrm{CA}}$ & CB & $\mathrm{S}$ & $\mathrm{H}$ & & 0.00 & 308.10 & & & \\
\hline $\mathrm{CA}$ & $\mathrm{CB}$ & $\mathrm{S}$ & $\mathrm{CA}$ & & 0.00 & 493.02 & & & \\
\hline $\mathrm{CB}$ & CB & $\mathrm{CA}$ & CR & & 0.00 & 540.59 & & & \\
\hline
\end{tabular}




\section{S1.7 FF-7 by Alexiadis and Mavrantzas ${ }^{8}$ based on DREIDING9}

The functional form of FF-7 is

$$
\begin{aligned}
& U=\sum_{\text {bonds }} \frac{k_{r}}{2}\left(r-r_{0}\right)^{2}+\sum_{\text {angles }} \frac{k_{\theta}}{2}\left(\theta-\theta_{0}\right)^{2}+\sum_{\text {dihedrals }} \sum_{n=1}^{k} k_{n}\left(1+\cos \left(n \phi-\phi_{n}\right)\right) \\
& +\sum_{\text {impropers }} \frac{K_{\xi}}{2}\left(\xi-\xi_{0}\right)^{2}+ \\
& \sum_{\text {nonbonded }}\left\{4 \varepsilon_{i j}\left[\left(\frac{\sigma_{i j}}{r_{i j}}\right)^{12}-\left(\frac{\sigma_{i j}}{r_{i j}}\right)^{6}\right]+\frac{C q_{i} q_{j}}{\varepsilon_{r} r_{i j}}\right\}
\end{aligned}
$$

The coding of atom types for RR-P3HT and the values of the force field parameters for the non-bonded and bonded interactions in FF-7 are presented in Table S1.7. 
Table S1.7. Potential form and parameters of FF-7.

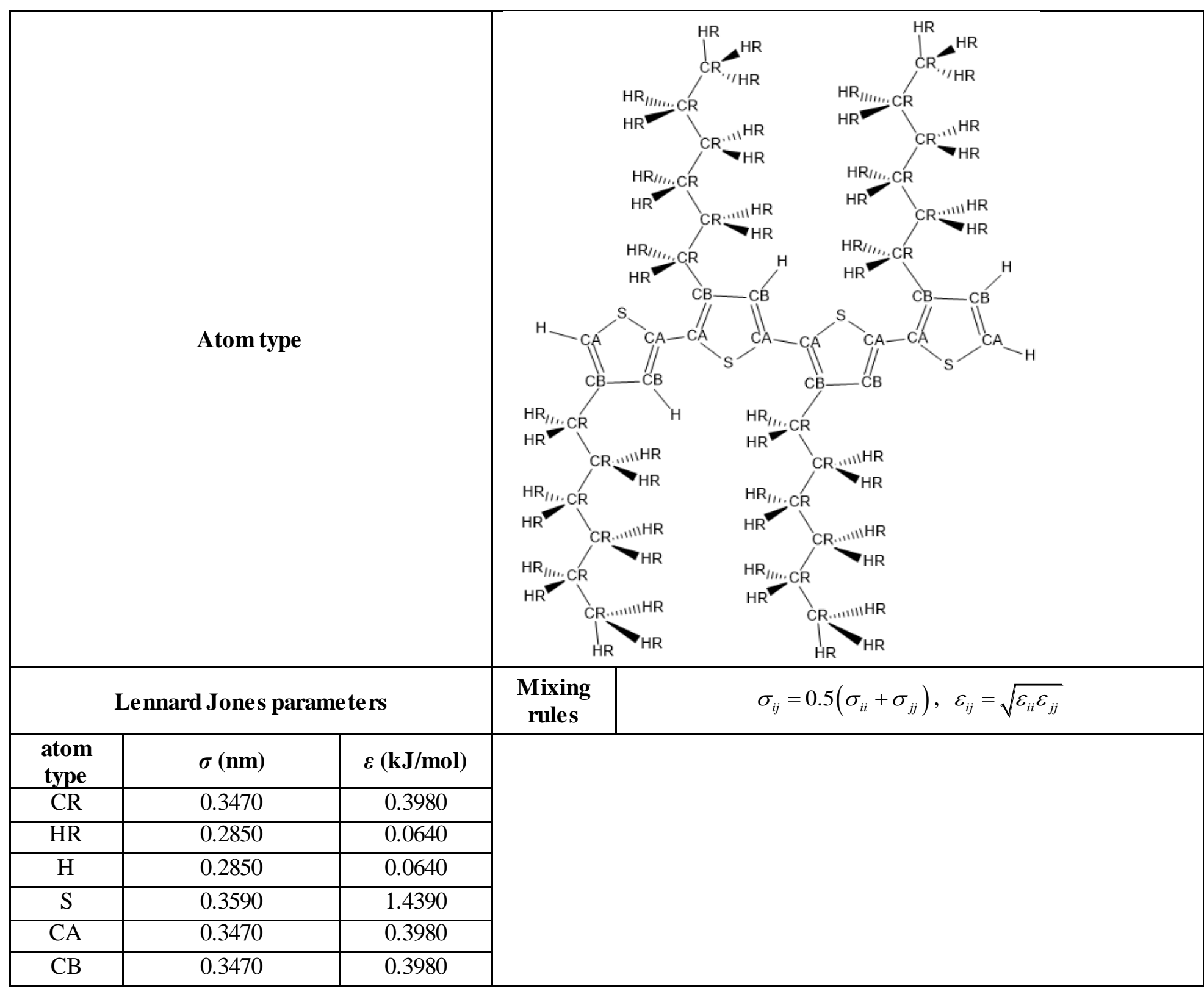




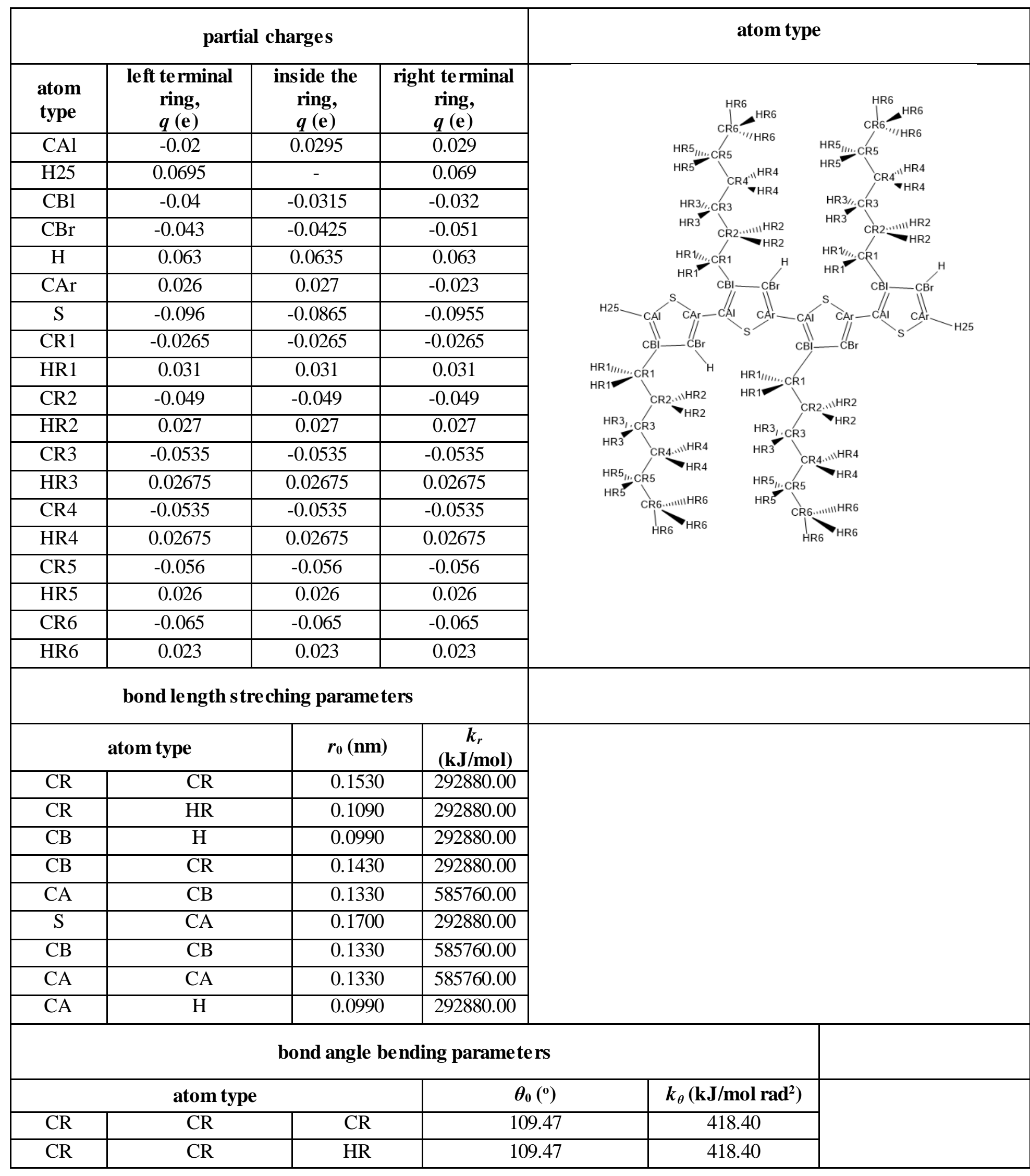




\begin{tabular}{|c|c|c|c|c|}
\hline HR & CR & HR & 109.47 & 418.40 \\
\hline CR & CR & CB & 109.47 & 418.40 \\
\hline HR & CR & CB & 109.47 & 418.40 \\
\hline CR & CB & CB & 120.00 & 418.40 \\
\hline CR & CB & CA & 120.00 & 418.40 \\
\hline CB & CB & CA & 120.00 & 418.40 \\
\hline H & CB & CB & 120.00 & 418.40 \\
\hline H & CB & CA & 120.00 & 418.40 \\
\hline CA & S & CA & 92.40 & 418.40 \\
\hline CA & CA & CB & 120.00 & 418.40 \\
\hline S & CA & CA & 120.00 & 418.40 \\
\hline S & CA & CB & 120.00 & 418.40 \\
\hline H & CA & S & 120.00 & 418.40 \\
\hline H & CA & CB & 120.00 & 418.40 \\
\hline
\end{tabular}

\section{dihedral parameters}

\begin{tabular}{|c|c|c|c|c|c|c|}
\hline \multicolumn{4}{|c|}{ atom type } & $\phi_{n}\left({ }^{0}\right)$ & $k_{n}(\mathrm{~kJ} / \mathrm{mol})$ & $n$ \\
\hline CR & CR & CR & CR & 0.00 & 0.465 & 3 \\
\hline CR & $\mathrm{CR}$ & CR & HR & 0.00 & 0.465 & 3 \\
\hline HR & $\mathrm{CR}$ & CR & HR & 0.00 & 0.000 & 0 \\
\hline HR & $\mathrm{CR}$ & CR & $\mathrm{CB}$ & 0.00 & 0.465 & 3 \\
\hline CR & $\mathrm{CR}$ & CR & $\mathrm{CB}$ & 0.00 & 0.465 & 3 \\
\hline HR & $\mathrm{CR}$ & $\mathrm{CB}$ & $\mathrm{CB}$ & -180.00 & 0.349 & 6 \\
\hline HR & $\mathrm{CR}$ & $\mathrm{CB}$ & $\mathrm{CA}$ & -180.00 & 0.349 & 6 \\
\hline CR & $\mathrm{CR}$ & $\mathrm{CB}$ & $\mathrm{CB}$ & -180.00 & 0.349 & 6 \\
\hline $\mathrm{CA}$ & $\mathrm{CB}$ & CR & CR & -180.00 & 0.349 & 6 \\
\hline $\mathrm{S}$ & $\mathrm{CA}$ & $\mathrm{CA}$ & $\mathrm{CB}$ & -180.00 & 5.230 & 2 \\
\hline $\mathrm{CB}$ & $\mathrm{CA}$ & $\mathrm{CA}$ & $\mathrm{CB}$ & -180.00 & 5.230 & 2 \\
\hline $\mathrm{S}$ & $\mathrm{CA}$ & $\mathrm{CA}$ & $\mathrm{S}$ & -180.00 & 5.230 & 2 \\
\hline $\mathrm{CA}$ & $\mathrm{CB}$ & $\mathrm{CB}$ & $\mathrm{CA}$ & -180.00 & 13.075 & 2 \\
\hline $\mathrm{H}$ & CB & $\mathrm{CB}$ & $\mathrm{CA}$ & -180.00 & 13.075 & 2 \\
\hline $\mathrm{H}$ & $\mathrm{CB}$ & $\mathrm{CB}$ & CR & -180.00 & 13.075 & 2 \\
\hline $\mathrm{CA}$ & CB & $\mathrm{CB}$ & $\mathrm{CR}$ & -180.00 & 13.075 & 2 \\
\hline$\overline{\mathrm{CA}}$ & $\mathrm{S}$ & $\mathrm{CA}$ & $\mathrm{CB}$ & -180.00 & 0.697 & 2 \\
\hline $\mathrm{CA}$ & $\mathrm{CB}$ & $\mathrm{CB}$ & $\mathrm{CA}$ & -180.00 & 13.075 & 2 \\
\hline$\overline{\mathrm{CB}}$ & $\mathrm{CB}$ & $\overline{\mathrm{CA}}$ & $\bar{S}$ & -180.00 & 13.075 & 2 \\
\hline $\mathrm{H}$ & $\mathrm{CA}$ & $\mathrm{S}$ & $\overline{\mathrm{CA}}$ & -180.00 & 0.697 & 2 \\
\hline $\mathrm{H}$ & $\mathrm{CA}$ & $\mathrm{CB}$ & $\mathrm{CB}$ & -180.00 & 13.075 & 2 \\
\hline $\mathrm{H}$ & $\mathrm{CA}$ & $\mathrm{CB}$ & $\mathrm{CR}$ & -180.00 & 13.075 & 2 \\
\hline CB & CB & $\mathrm{CA}$ & $\mathrm{CA}$ & -180.00 & 13.075 & 2 \\
\hline $\mathrm{CA}$ & $S$ & $\mathrm{CA}$ & $\mathrm{CA}$ & -180.00 & 0.697 & 2 \\
\hline
\end{tabular}




\begin{tabular}{|c|c|c|c|c|c|c|}
\hline $\mathrm{H}$ & $\overline{\mathrm{CB}}$ & $\mathrm{CA}$ & $\bar{S}$ & -180.00 & 13.075 & 2 \\
\hline $\mathrm{H}$ & $\mathrm{CB}$ & $\mathrm{CA}$ & $\mathrm{CA}$ & -180.00 & 13.075 & 2 \\
\hline $\mathrm{CA}$ & $\overline{\mathrm{CA}}$ & $\mathrm{CB}$ & $\overline{\mathrm{CR}}$ & -180.00 & 13.075 & 2 \\
\hline$\overline{\mathrm{CA}}$ & $\overline{\mathrm{CA}}$ & $\bar{S}$ & $\overline{\mathrm{CA}}$ & -180.00 & 0.697 & 2 \\
\hline $\mathrm{S}$ & $\mathrm{CA}$ & $\mathrm{CB}$ & $\mathrm{CR}$ & -180.00 & 13.075 & 2 \\
\hline $\mathrm{H}$ & $\overline{\mathrm{CB}}$ & $\mathrm{CA}$ & $\mathrm{H}$ & -180.00 & 13.075 & 2 \\
\hline \multicolumn{7}{|c|}{ improper parame ters } \\
\hline \multicolumn{4}{|c|}{ atom type } & $\xi_{0}\left({ }^{\circ}\right)$ & \multicolumn{2}{|c|}{$k_{\xi}(\mathbf{k J} / \mathbf{m o l})$} \\
\hline $\mathrm{CA}$ & $\mathrm{CB}$ & $\mathrm{S}$ & $\mathrm{H}$ & 0.0 & \multicolumn{2}{|c|}{167.4} \\
\hline $\mathrm{CB}$ & $\mathrm{CB}$ & $\mathrm{CA}$ & $\mathrm{H}$ & 0.0 & \multicolumn{2}{|c|}{167.4} \\
\hline $\mathrm{CA}$ & $\bar{S}$ & $\mathrm{CB}$ & $\overline{\mathrm{CA}}$ & 0.0 & \multicolumn{2}{|c|}{167.4} \\
\hline $\mathrm{CB}$ & $\mathrm{CB}$ & $\mathrm{CA}$ & $\overline{C R}$ & 0.0 & \multicolumn{2}{|c|}{167.4} \\
\hline
\end{tabular}


S2. Comparison of the seven force fields

\section{A. Orientational relaxation of the 20-P3HT oligomer}

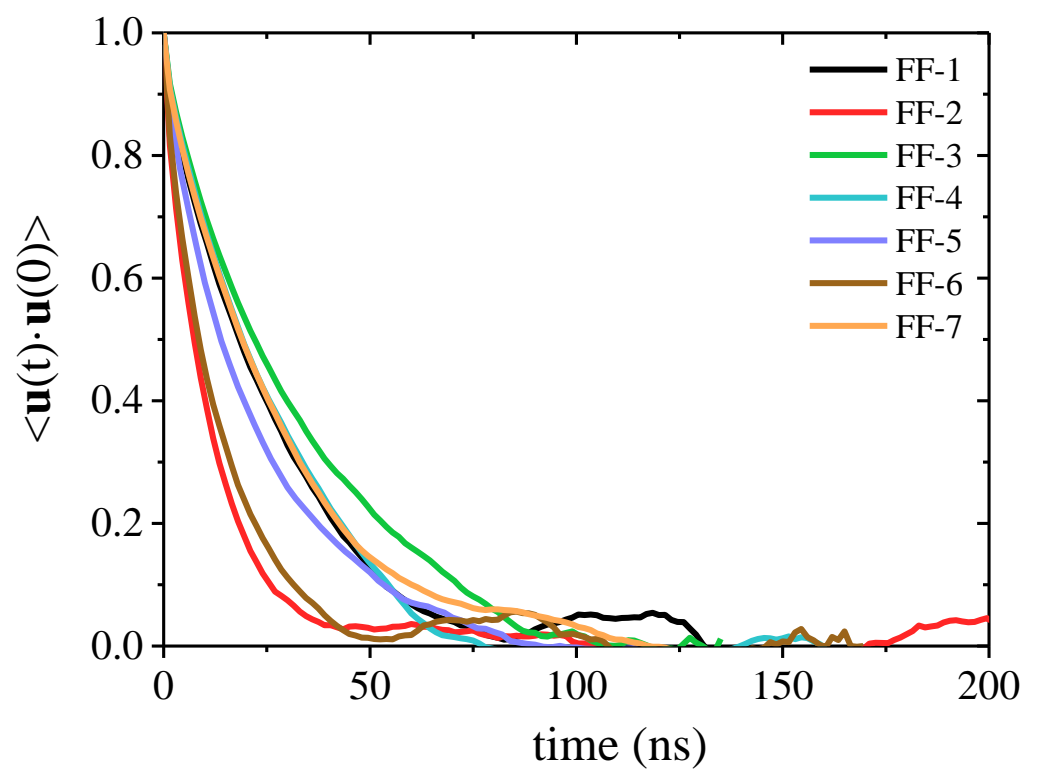

Figure S2.1. MD predictions for the rate of decay of the $\langle\mathbf{u}(t) \cdot \mathbf{u}(0)\rangle$ autocorrelation function for the 20P3HT system. Results are shown for all seven FFs examined $(T=650 \mathrm{~K}, P=1 \mathrm{~atm})$. 
B. Inter-molecular pair correlation functions of thiophene rings' centers-of-mass for the 20-P3HT oligomer

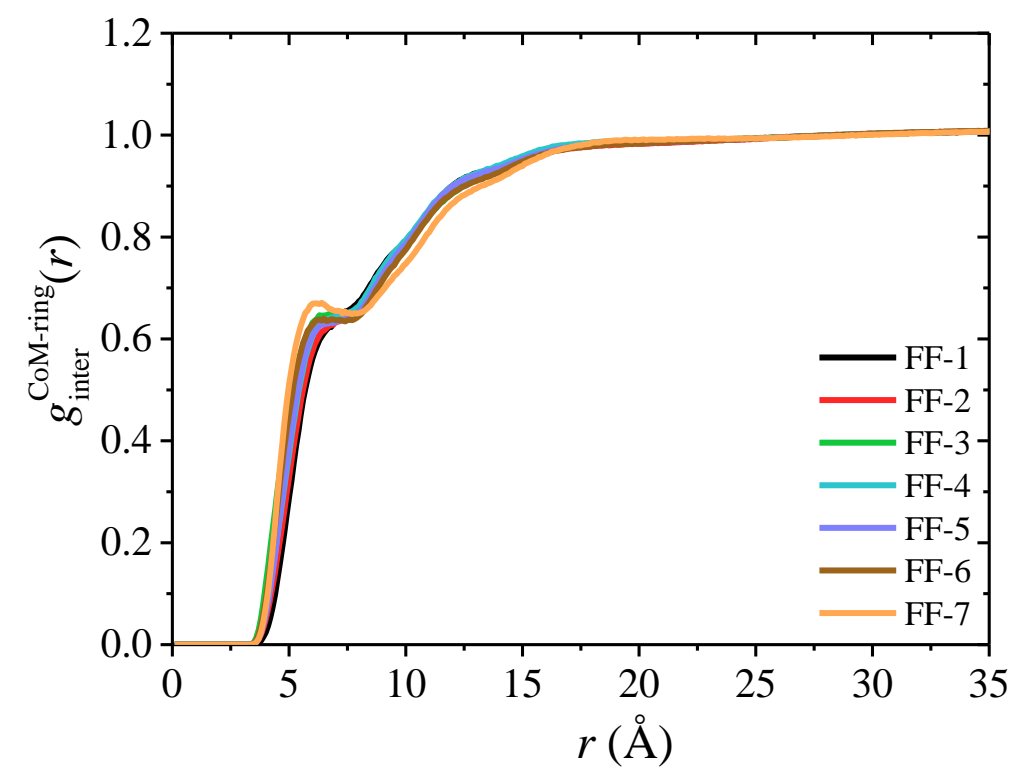

Figure S2.2. MD simulation results for the inter-molecular radial distribution function $g_{\text {inter }}^{\text {CoM-ring }}(r)$ of the thiophene rings' centers-of-mass $(T=650 \mathrm{~K}, P=1 \mathrm{~atm})$. 


\section{Density versus simulation time}

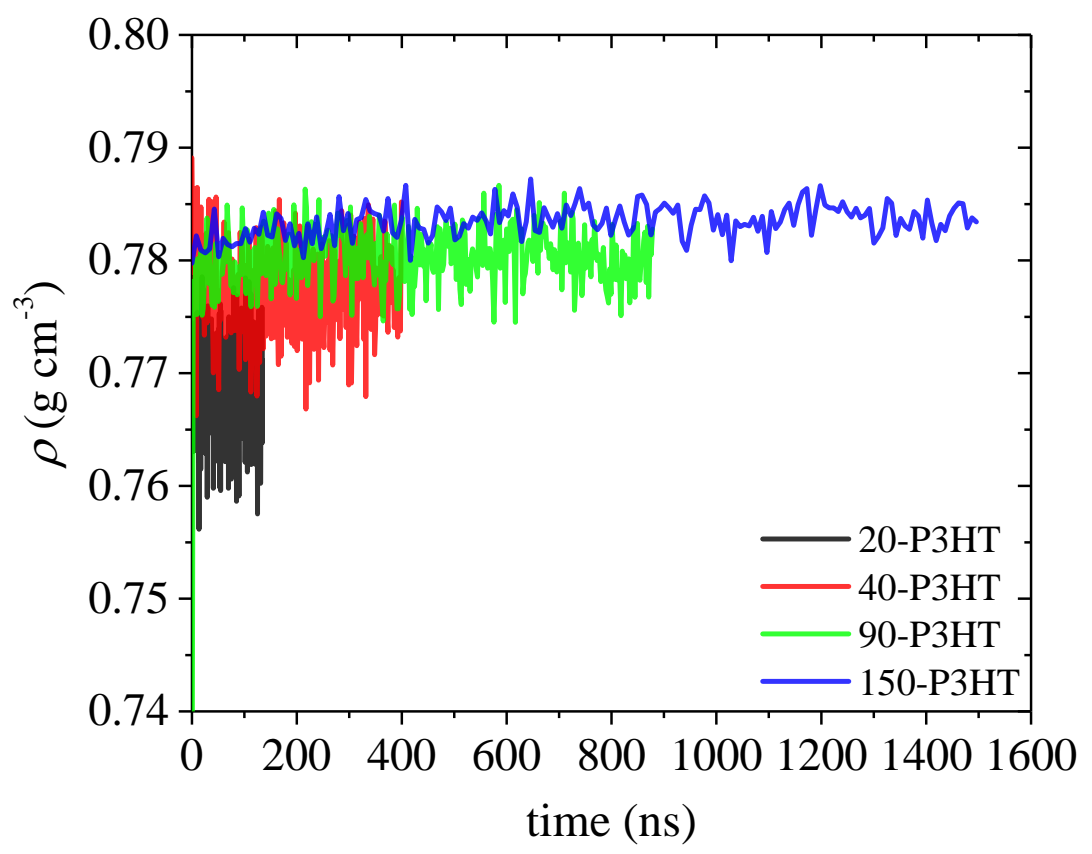

Figure S2.3. Time evolution of the instantaneous value of the density $\rho$ of the $n$-P3HT melts $(T=700 \mathrm{~K}, P$ $=1 \mathrm{~atm})$ with $n=20,40,90$, and 150 monomers per chain, as obtained from the MD simulations with FF6. 


\section{Distributions of the key dihedral angles}

The distributions of the key dihedral angles for all seven FFs examined are presented in Figures S2.5S2.8. The results have been obtained with the 20 -P3HT system $(T=650 \mathrm{~K}, P=1 \mathrm{~atm})$. The coding of angles is based on the atom type assignment shown in Figure S2.1 below.

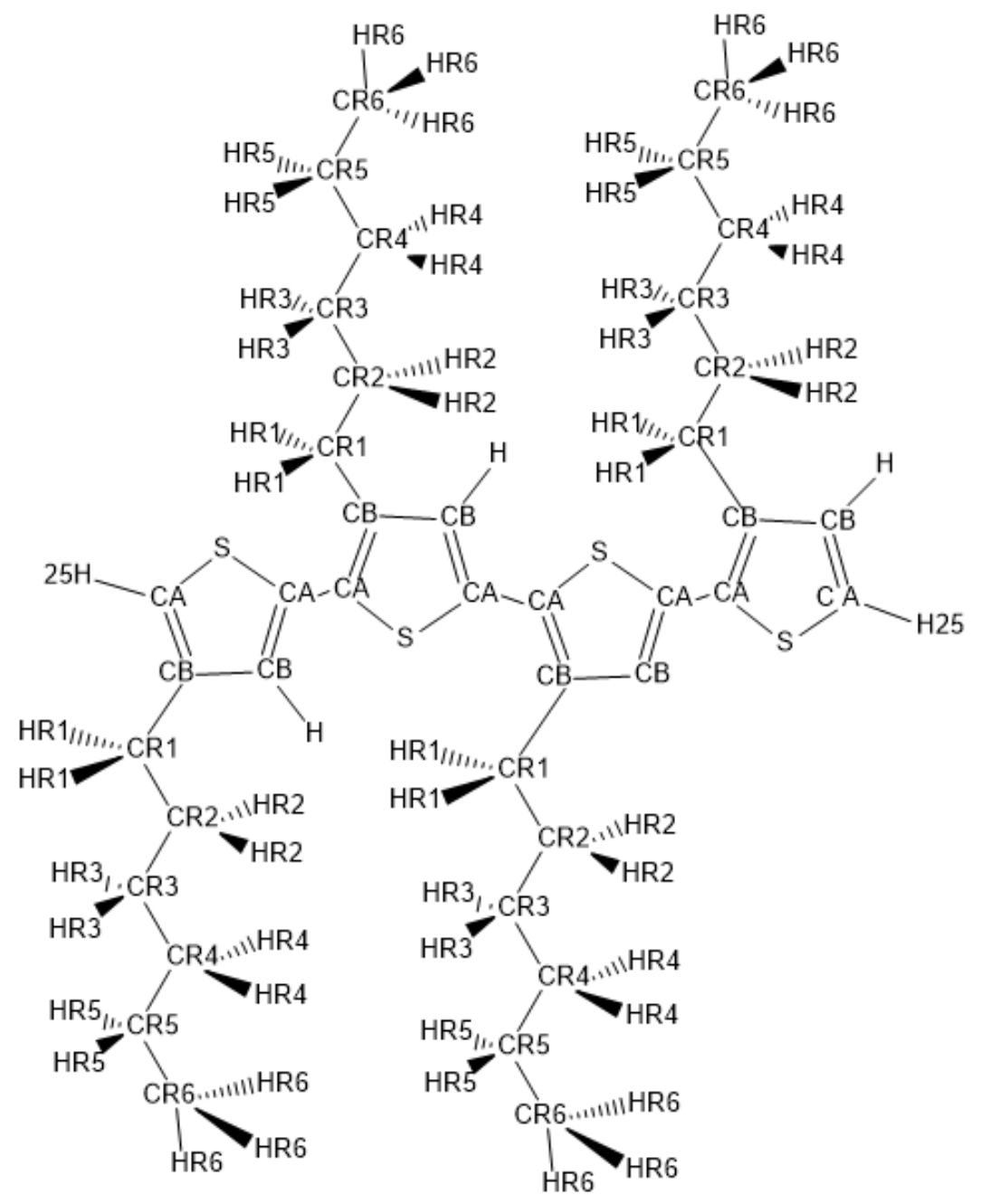

Figure S2.4. Assignment of atom types in a regioregular 4-P3HT chain. 

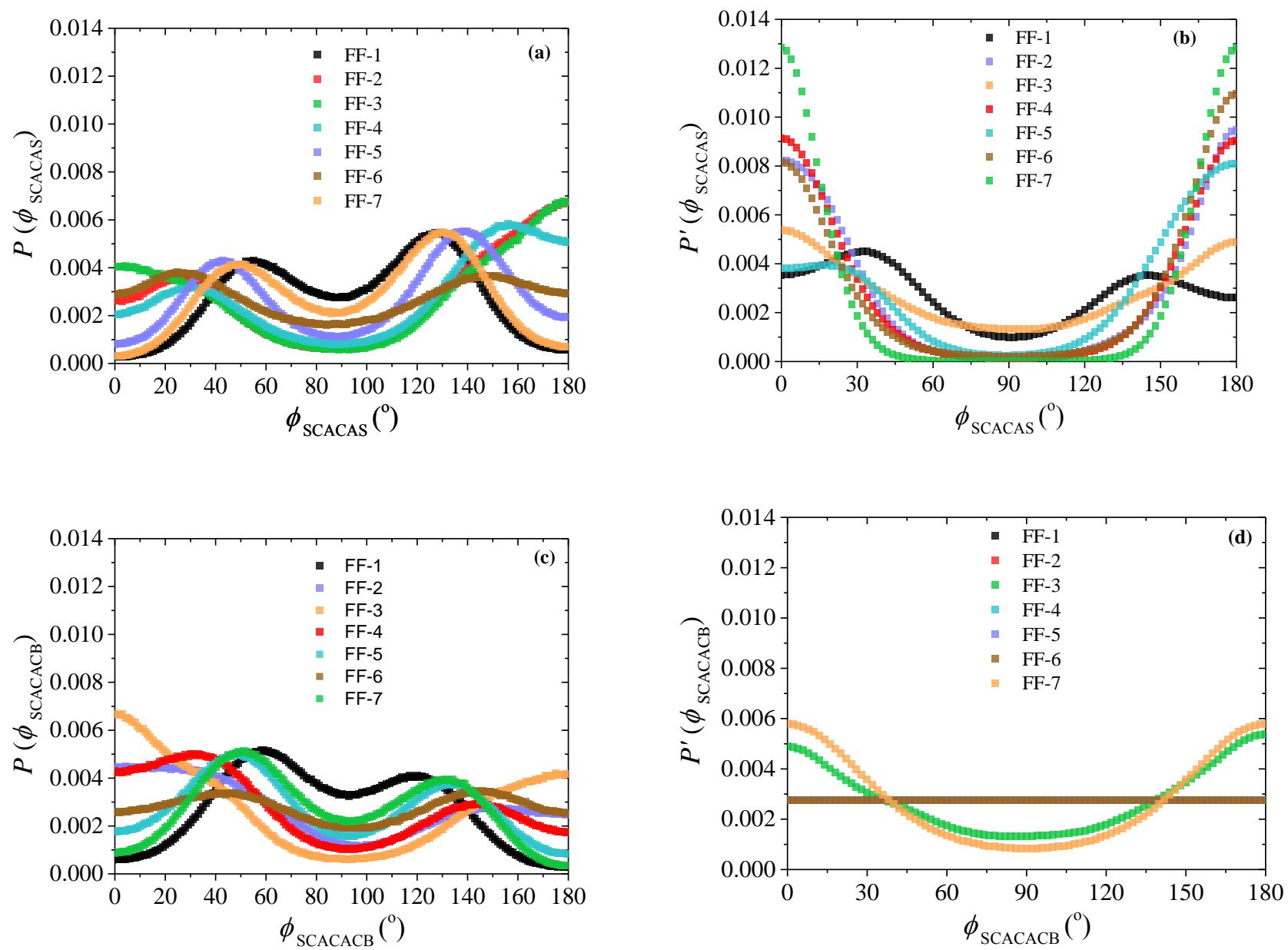

Figure S2.5. (a) Distributions of the inter-ring SCACAS dihedral for the various FFs examined, and (b) the corresponding Boltzmann probability density functions. (c) Same as with (a) but for the inter-ring SCACACB dihedral, and (d) the corresponding Boltzmann probability density functions. For the coding of atom types, see Figure S2.4. Only half of the horizontal axis is shown due to the symmetry of curves. 

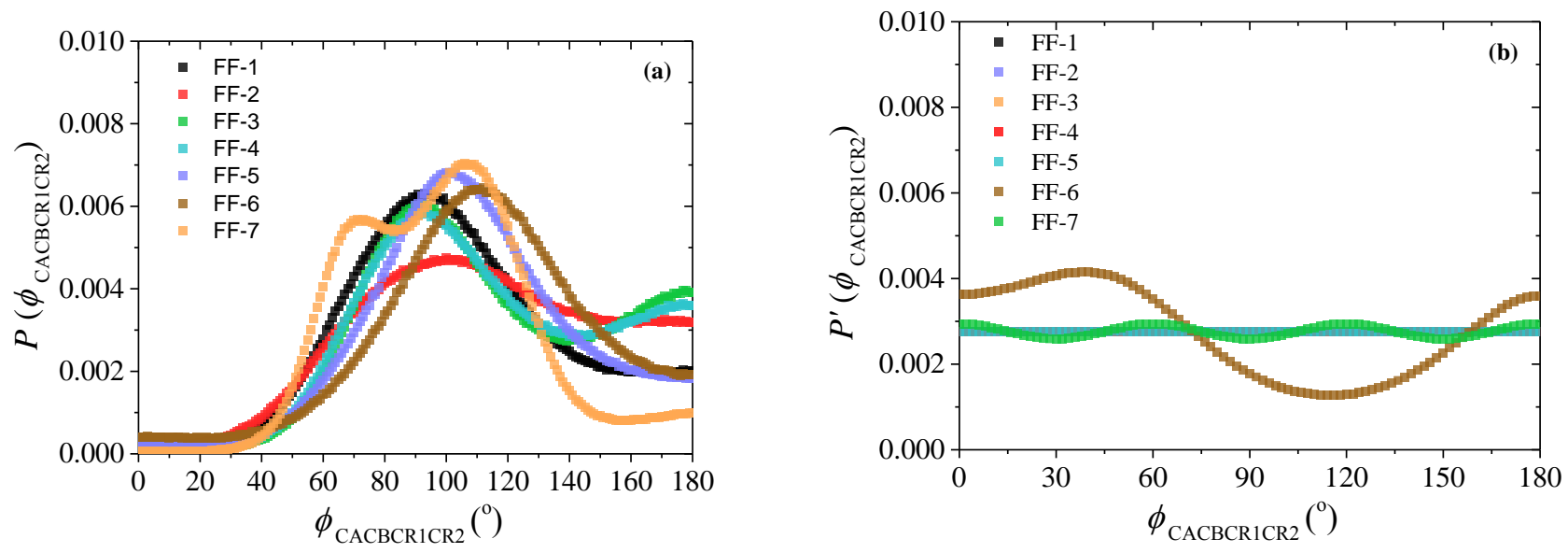

Figure S2.6. (a) Distributions of the ring-side chain CACBCR1CR2 dihedral for the various FFs examined, and (b) the corresponding Boltzmann probability density functions. For the coding of atom types, see Figure S2.4. Only the half of the horizontal axis is shown due to the symmetry of curves.
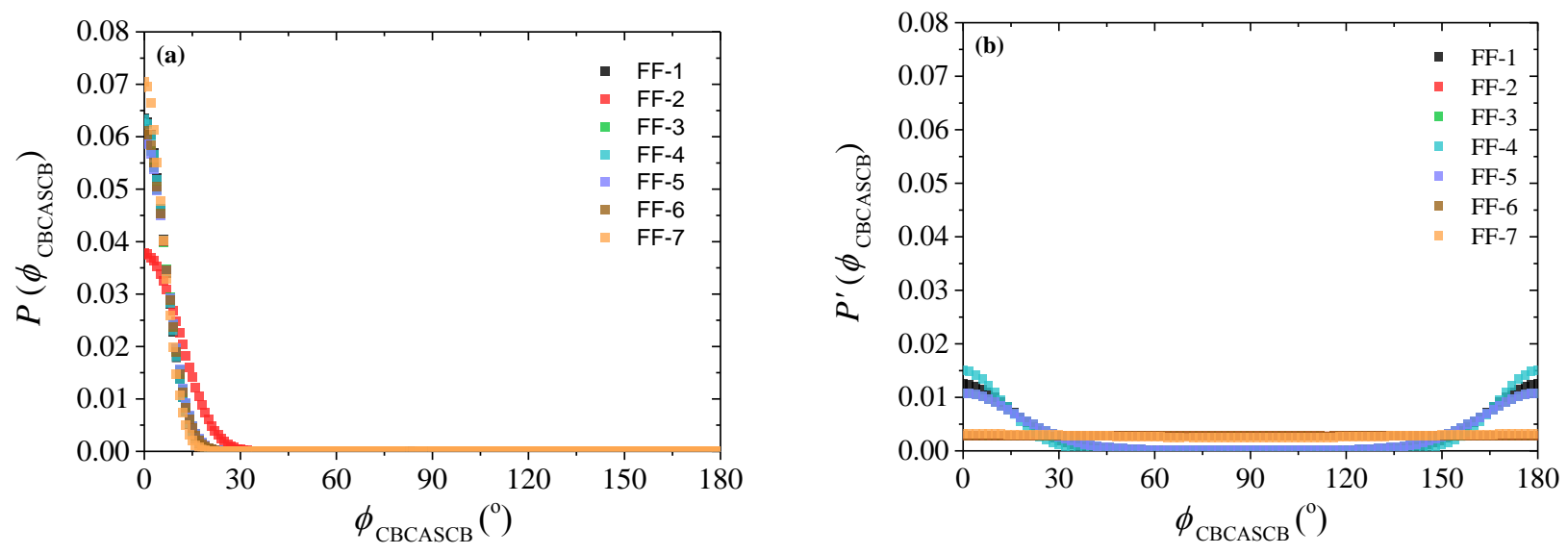

Figure S2.7. Same as with Figure S2.6 but for the intra-ring CBCASCB dihedral. 

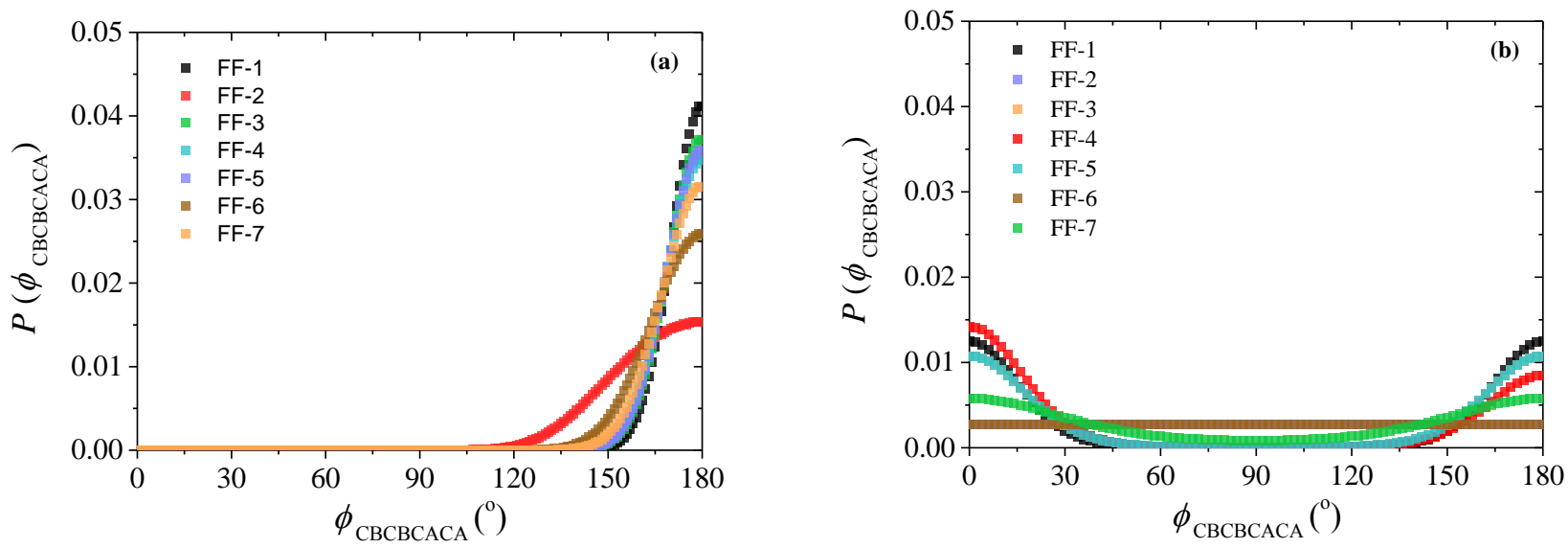

Figure S2.8. Same as with Figure S2.6 but for the intra-ring CBCBCACA dihedral.

Significant differences are observed in the inter-ring dihedral distributions displayed in Figure $\mathrm{S} 2.5$ between the various FFs. In particular, the presence of significant peaks between $0^{\circ}$ (cis) and $180^{\circ}$ (trans) in all of them are signs of deviations from the planar configuration. In contrast, the intra-ring dihedral distributions are quite similar, which demonstrates the limited conformational freedom of the thiophene rings. For example, in Figure S2.7 only very small deviations from planarity (the cis conformation) are observed. The same is observed for the distribution of the CBCBCACA dihedral angle associated with ring bending, where deviations from the trans conformation for all FFs are less than $40^{\circ}$, except for FF-2.

We also see that in most cases the dihedral angle distributions obtained from MD are quite different from the corresponding theoretical ones (from the Boltzmann distribution of the corresponding torsion energy) which should be attributed to strong non-bonded (i.e., van der Waals and Coulomb) interactions. 


\section{E. Intra-molecular pair correlation functions of thiophene rings' centers-of-mass}

We have also calculated the intra-molecular radial pair correlation functions of the centers-of-mass $(\mathrm{CoM})$ of thiophene rings, and the results are reported in Figure S2.9. They refer again to the 20-P3HT system $(T=650 \mathrm{~K}, P=1 \mathrm{~atm})$. For all FFs examined, the curves present peaks at almost the same distances $(\sim 4 \AA, \sim 7.7 \AA$, and $\sim 11.5 \AA)$, something that should have been expected given that in all these FFs the van der Waals parameters $(\sigma$ and $\varepsilon)$ are practically the same. ${ }^{10,11}$

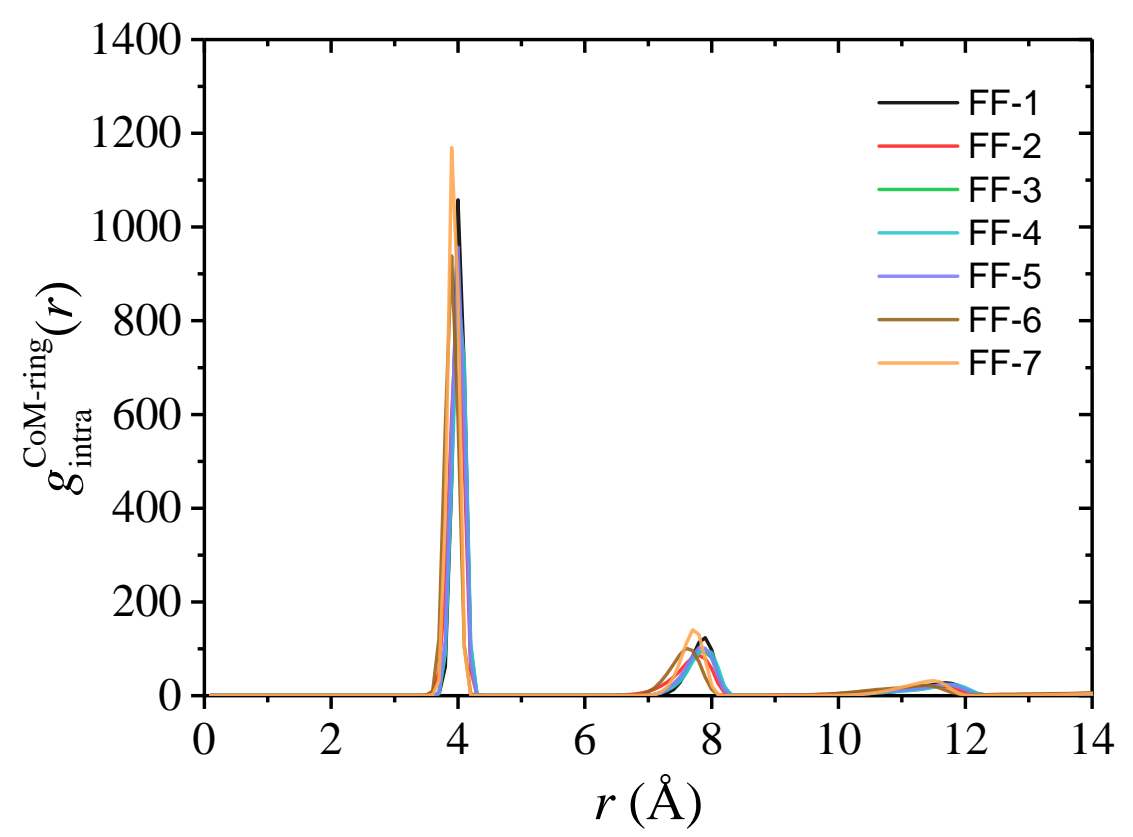

Figure S2.9. MD predictions for the intra-molecular radial pair correlation function of thiophene rings' centers-of-mass from the various FFs. 


\section{F. Worm Like Chain (WLC) model predictions}

Table S2.1. Numerical results from the WLC model for the quantities $\left\langle R^{2}\right\rangle$ and $\left\langle R_{\mathrm{g}}^{2}\right\rangle$, and their ratio using eqs 2 and 3 in the main manuscript. The data refer to the 20-P3HT melt and are shown at various temperatures $(P=1 \mathrm{~atm}) .\left\langle R_{\mathrm{g}}^{2}\right\rangle_{\text {chain }}$ denotes the mean-square radius-of-gyration of the entire chain as calculated directly from our MD simulations.

\begin{tabular}{|c|c|c|c|c|c|c|c|}
\hline $\bar{T} \overline{T(\mathbf{K})}$ & FF-1 & FF-2 & FF-3 & FF-4 & FF-5 & FF-6 & FF-7 \\
\hline & \multicolumn{7}{|c|}{$\left\langle R_{\mathrm{g}}^{2}\right\rangle_{\mathrm{WLC}}\left(\AA^{2}\right)$} \\
\hline 700 & 312 & 280 & 315 & 330 & 303 & 265 & 313 \\
\hline 650 & 319 & 279 & 317 & 335 & 307 & 273 & 314 \\
\hline 550 & 324 & 294 & 311 & 339 & 311 & 273 & 327 \\
\hline \multirow[t]{2}{*}{500} & - & - & - & - & - & 274 & - \\
\hline & \multicolumn{7}{|c|}{$\left\langle R^{2}\right\rangle_{\mathrm{WLC}}\left(\AA^{2}\right)$} \\
\hline 700 & 2783 & 2405 & 2827 & 3018 & 2678 & 2258 & 2854 \\
\hline 650 & 2871 & 2383 & 2846 & 3090 & 2731 & 2347 & 2871 \\
\hline 550 & 2940 & 2563 & 2773 & 3145 & 2776 & 2353 & 3041 \\
\hline 500 & - & - & - & - & - & 2364 & - \\
\hline & 0 & & & & & & \\
\hline 700 & 8.9 & 8.6 & 9.0 & 9.2 & 8.8 & 8.5 & 9.1 \\
\hline 650 & 9.0 & 8.6 & 9.0 & 9.2 & 8.9 & 8.6 & 9.1 \\
\hline 550 & 9.1 & 8.7 & 8.9 & 9.3 & 8.9 & 8.6 & 9.3 \\
\hline \multirow[t]{2}{*}{500} & - & - & - & - & - & 8.6 & - \\
\hline & \multicolumn{7}{|c|}{$\left\langle R_{\mathrm{g}}^{2}\right\rangle_{\text {chain }}\left(\AA^{2}\right)$} \\
\hline 700 & $385 \pm 3$ & $344 \pm 3$ & $387 \pm 2$ & $399 \pm 2$ & $380 \pm 2$ & $341 \pm 3$ & $388 \pm 4$ \\
\hline 650 & $393 \pm 6$ & $345 \pm 1$ & $387 \pm 6$ & $404 \pm 4$ & $384 \pm 5$ & $346 \pm 1$ & $391 \pm 3$ \\
\hline 550 & $402 \pm 3$ & $361 \pm 2$ & $395 \pm 5$ & $412 \pm 2$ & $389 \pm 6$ & $350 \pm 3$ & $402 \pm 4$ \\
\hline 500 & - & - & - & - & - & $352 \pm 2$ & - \\
\hline
\end{tabular}


Table S2.2. Same as with Table S2.1 but for the 40-P3HT melt.

\begin{tabular}{|c|c|c|c|c|c|c|c|}
\hline$T(\mathbf{K})$ & FF-1 & FF-2 & FF-3 & FF-4 & FF-5 & FF-6 & FF-7 \\
\hline & \multicolumn{7}{|c|}{$\overline{\left\langle R_{g}^{2}\right\rangle_{\mathrm{wLC}}\left(\AA^{2}\right)}$} \\
\hline 700 & 873 & 762 & 903 & 965 & 874 & 733 & 903 \\
\hline 650 & 875 & 781 & 913 & 987 & 878 & 741 & 955 \\
\hline \multirow[t]{2}{*}{550} & - & - & - & - & - & 764 & - \\
\hline & \multicolumn{7}{|c|}{$\left\langle R^{2}\right\rangle_{\mathrm{WLC}}\left(\AA^{2}\right)$} \\
\hline 700 & 6805 & 5733 & 7096 & 7755 & 6814 & 5509 & 7205 \\
\hline 650 & 6820 & 5905 & 7200 & 7992 & 6856 & 5584 & 7764 \\
\hline $\mathbf{5 5 0}$ & - & - & - & & - & 5799 & - \\
\hline & \multicolumn{7}{|c|}{$\left\langle R^{2}\right\rangle_{\mathrm{wLC}} /\left\langle R_{\mathrm{g}}^{2}\right\rangle_{\mathrm{wLC}}$} \\
\hline 700 & 7.8 & 7.5 & 7.9 & 8.0 & 7.8 & 7.5 & 8.0 \\
\hline 650 & 7.8 & 7.6 & 7.9 & 8.1 & 7.8 & 7.5 & 8.1 \\
\hline \multirow[t]{2}{*}{550} & - & - & - & - & - & 7.6 & - \\
\hline & \multicolumn{7}{|c|}{$\left\langle R_{\mathrm{g}}^{2}\right\rangle_{\text {chain }}\left(\AA^{2}\right)$} \\
\hline 700 & $979 \pm 15$ & $843 \pm 6$ & $996 \pm 17$ & $1057 \pm 8$ & $955 \pm 12$ & $825 \pm 12$ & $1003 \pm 11$ \\
\hline 650 & $987 \pm 15$ & $864 \pm 12$ & $1007 \pm 17$ & $1072 \pm 12$ & $974 \pm 16$ & $827 \pm 10$ & $1052 \pm 14$ \\
\hline 550 & - & - & - & - & - & $871 \pm 6$ & \\
\hline
\end{tabular}

\section{G. Extrapolated densities at $300 \mathrm{~K}$}

Table S2.3. Predicted densities $\left(\mathrm{g} / \mathrm{cm}^{3}\right)$ at $300 \mathrm{~K}$ of the $20-\mathrm{P} 3 \mathrm{HT}$ and $40-\mathrm{P} 3 \mathrm{HT}$ systems from the various force fieds examined. The reported values were obtained by extrapolating the MD data from higher temperatures (Figure 3 of the main manuscript) down to $300 \mathrm{~K}$.

\begin{tabular}{|c|c|c|c|c|c|c|c|}
\hline $\bar{T} T(\mathbf{K})$ & FF-1 & FF-2 & FF-3 & FF-4 & FF-5 & FF-6 & FF-7 \\
\hline \multirow{3}{*}{300} & \multicolumn{7}{|c|}{ 20-P3HT } \\
\hline & 1.053 & 1.071 & 1.077 & 1.078 & 1.064 & 1.085 & 0.981 \\
\hline & \multicolumn{7}{|c|}{ 40-Р3НT } \\
\hline 300 & 1.061 & 1.074 & 1.097 & 1.087 & 1.070 & 1.092 & 0.989 \\
\hline
\end{tabular}




\section{S3. Additional Results from the MD Simulations with FF-6}

\section{A. Spatial extent of cis and trans segments}

The different conformation characteristics of cis and trans segments are better understood by looking at the corresponding conditional (at fixed length) probabilities of their end-to-end distances shown in Figure S3.1. The end-to-end distance of a cis segment is smaller than that of a trans segment of exactly the same length, and the difference increases with increasing length as trans segments are associated with more extended portions of the chain while cis segments are associated with turns and foldings along the chain backbone.

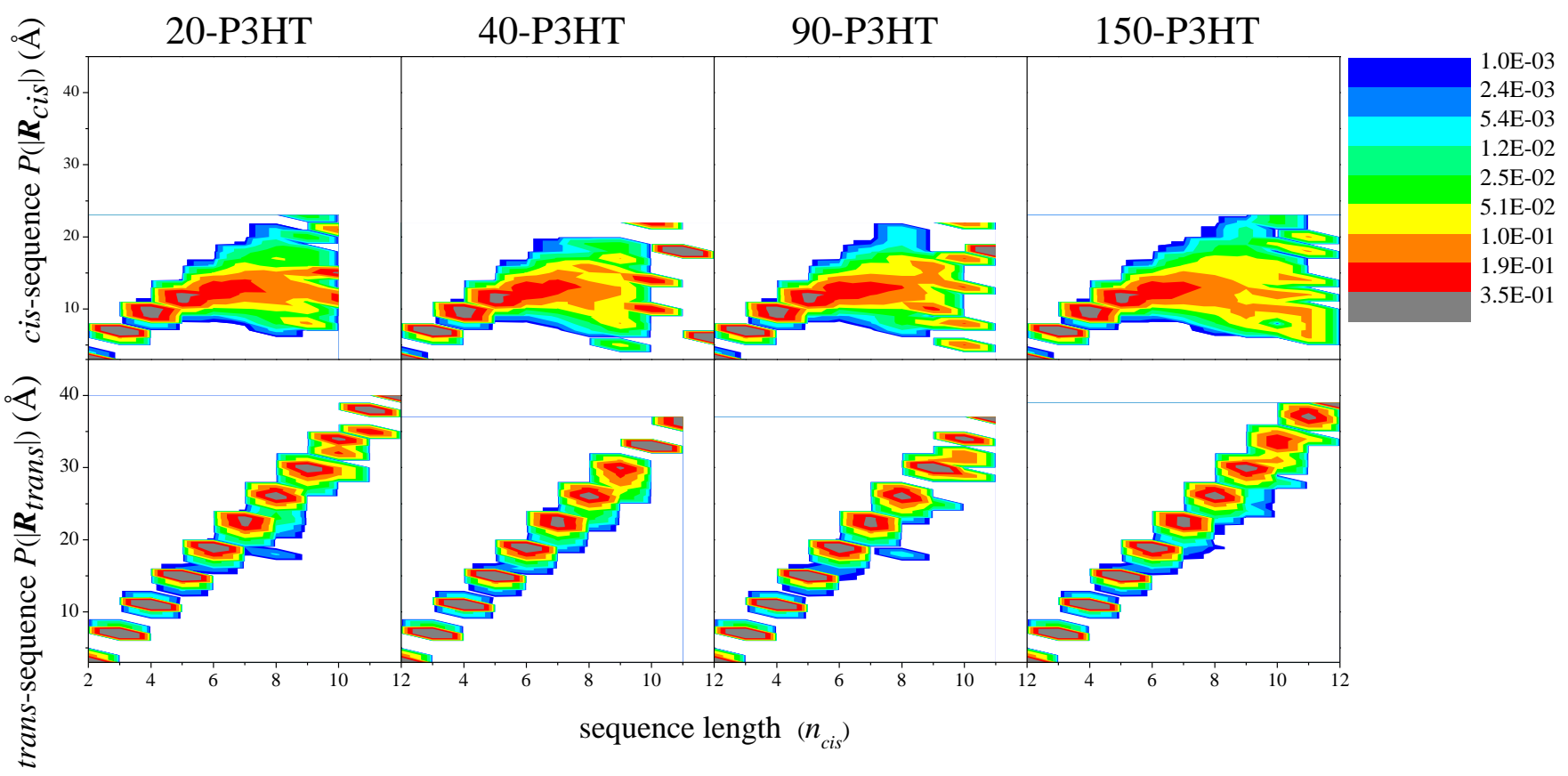

Figure S3.1. MD simulation predictions with FF-6 for the distribution of the length of cis (upper row) and trans (lower row) segments along the P3HT chain as a function of the segment length in monomer units, $n_{\text {cis. }}$ Results from the 20-P3HT, 40-P3HT, 90-P3HT, and 150-P3HT system at $T=700 \mathrm{~K}(P=1$ atm). 
B. Variation of the persistence length and conjugation length with molecular weight
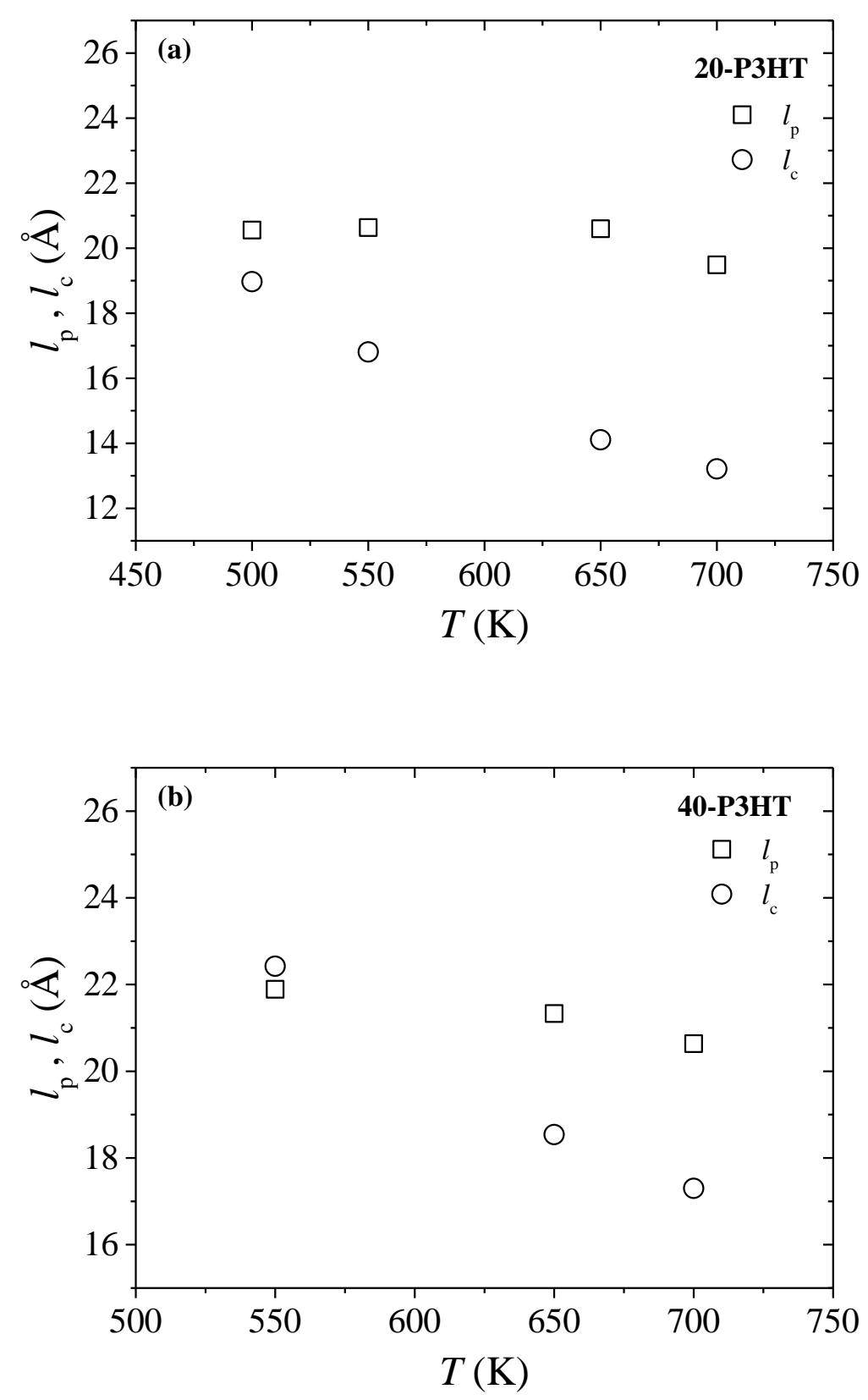


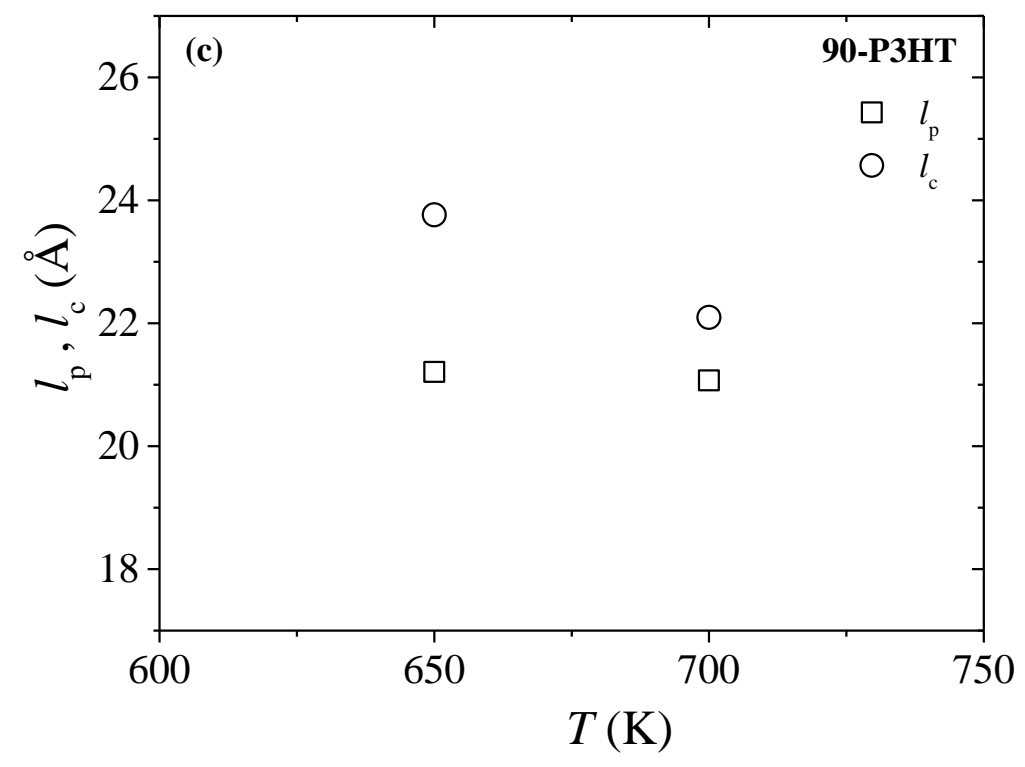

Figure S3.2. MD simulation predictions with FF-6 for the persistence length $l_{\mathrm{p}}$ and the effective conjugation length $l_{\mathrm{c}}$ of: (a) the 20-P3HT, (b) the 40-P3HT, and (c) the 90-P3HT system $(T=700 \mathrm{~K}, P$ $=1 \mathrm{~atm})$. The error bars are comparable to or smaller than the size of the symbols used to represent them and have been omitted for clarity. 
C. Temperature dependence of the distribution of the key dihedral angles from the MD simulations with FF-6
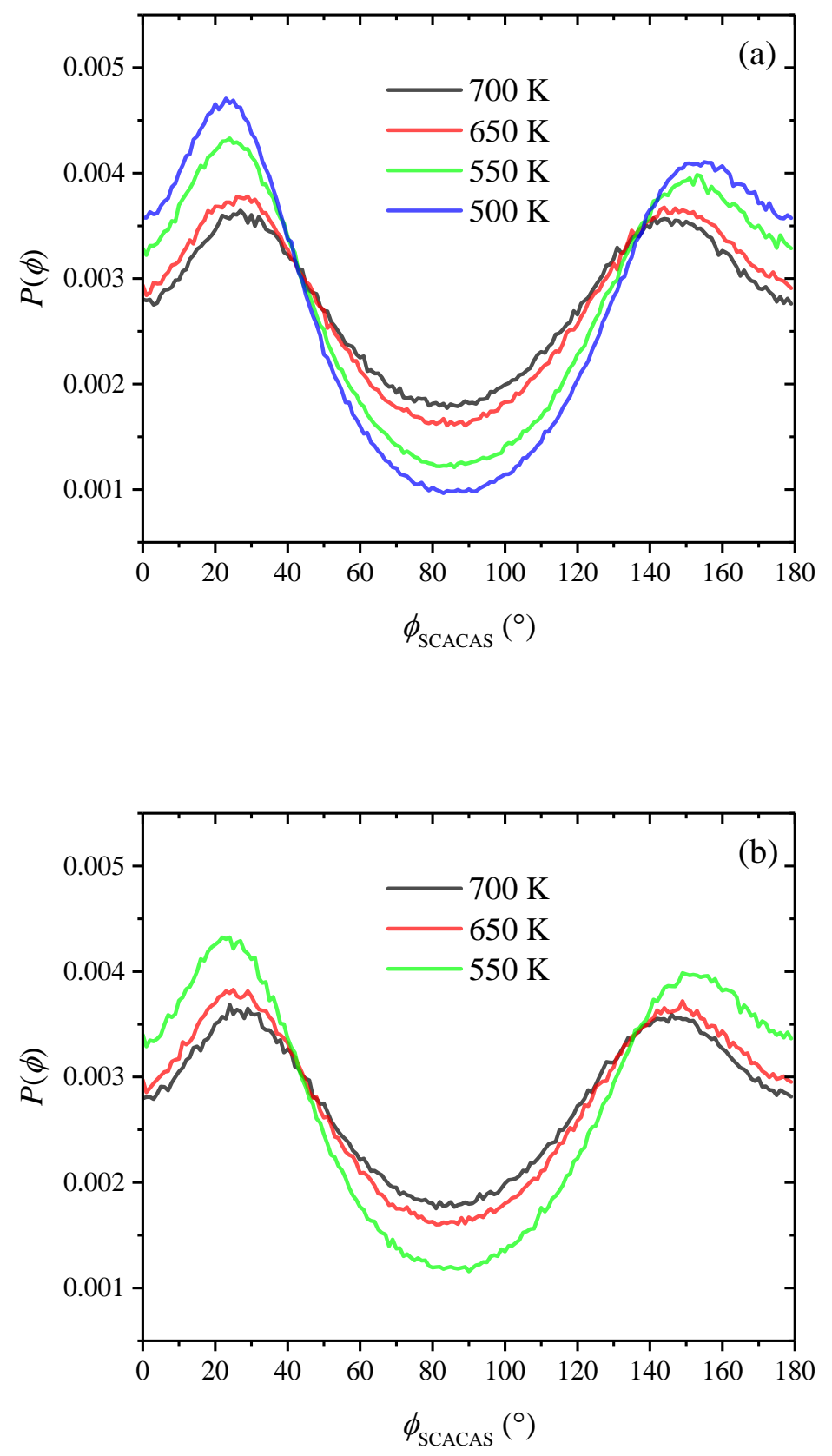


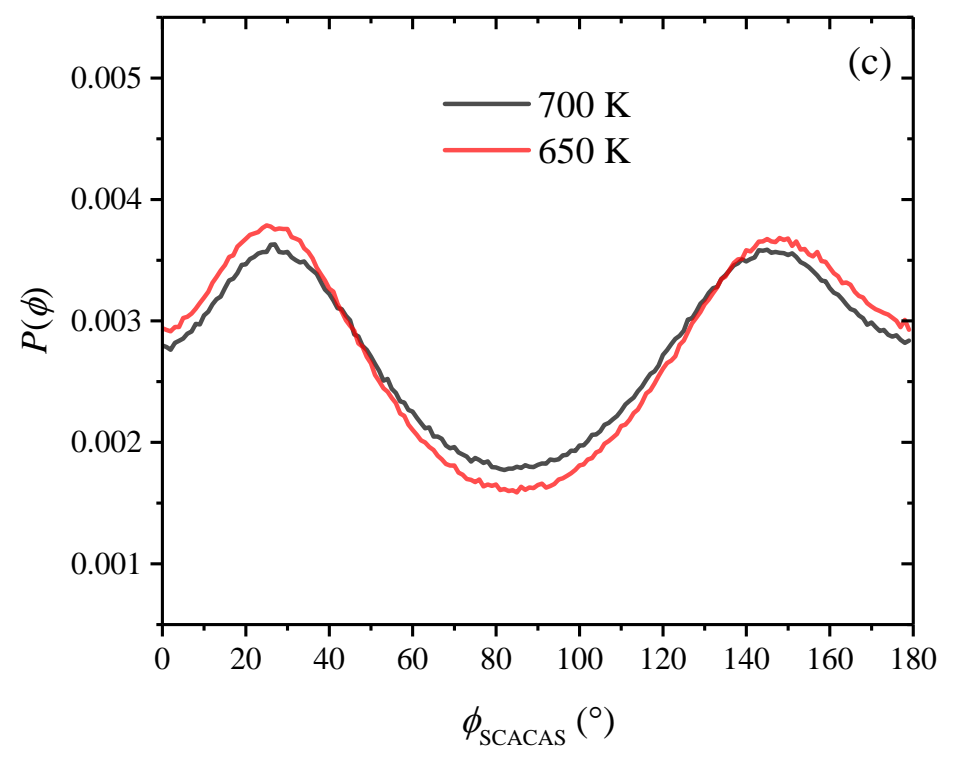

Figure S3.3. MD simulation predictions with FF-6 for the distribution of the inter-ring SCACAS dihedral as a function of temperature for: (a) the 20-P3HT system, (b) the 40-P3HT, and (c) the 90-P3HT system $(P=1 \mathrm{~atm})$. For the coding of atom types, see Figure S2.1. Only half of the horizontal axis is shown due to the symmetry of curves. 


\section{Estimation of the ECL based on the unweighted $n_{\mathrm{c}}$ distributions}

Another way to calculate $n_{\mathrm{c}}$ is to consider the length of the maximum conjugated segment along the chain but without multiplying it with the number of its occurrences in the chain. The results obtained through such a calculation agree with those based on the weighted LRH distributions (Figure S3.4) but are systematically higher by about half a backbone segment (Figure S3.5). However, this difference results in higher values of ECL $(\sim 1.9-2.0 \AA)$ and also causes a shift in the molecular weight where the ECL $\left(l_{\mathrm{p}}\right)$ becomes higher than the persistence length $\left(l_{\mathrm{p}}\right)$ from the 80 - to the 50 -P3HT chain (Figure S3.6).

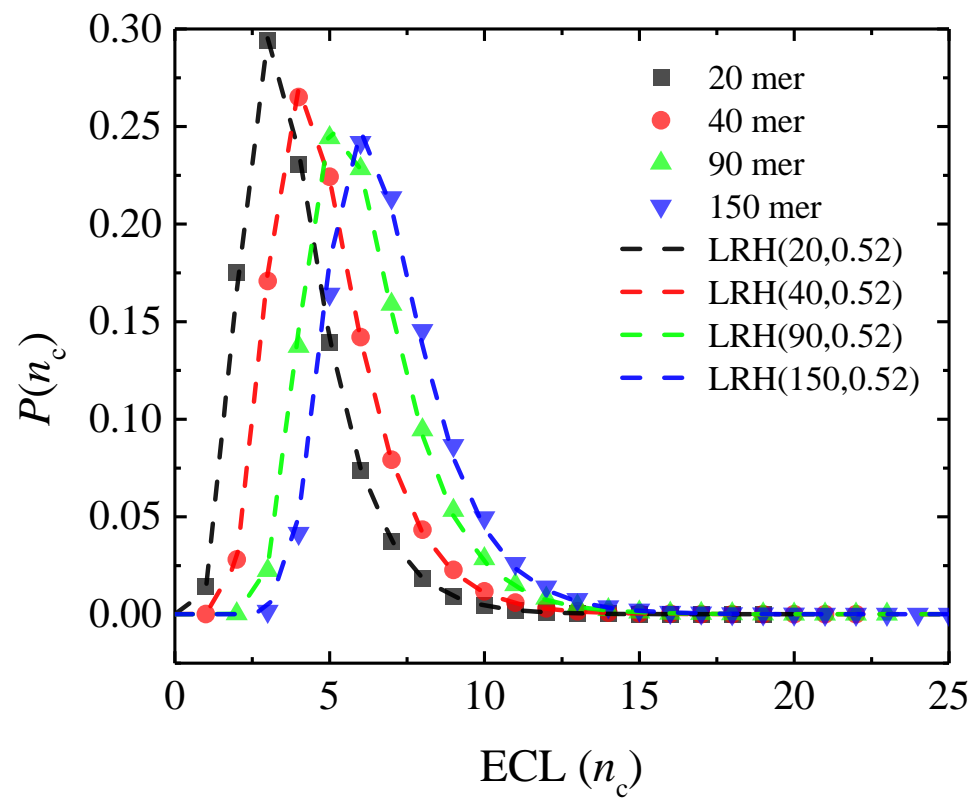

Figure S3.4. Probability distribution of the unweighted effective conjugation length in units of consecutive planar angles $n_{\mathrm{c}}$ for all $n$-P3HT systems studied (symbols) with the FF-6 force field $(T=700 \mathrm{~K}, P=1 \mathrm{~atm})$. 


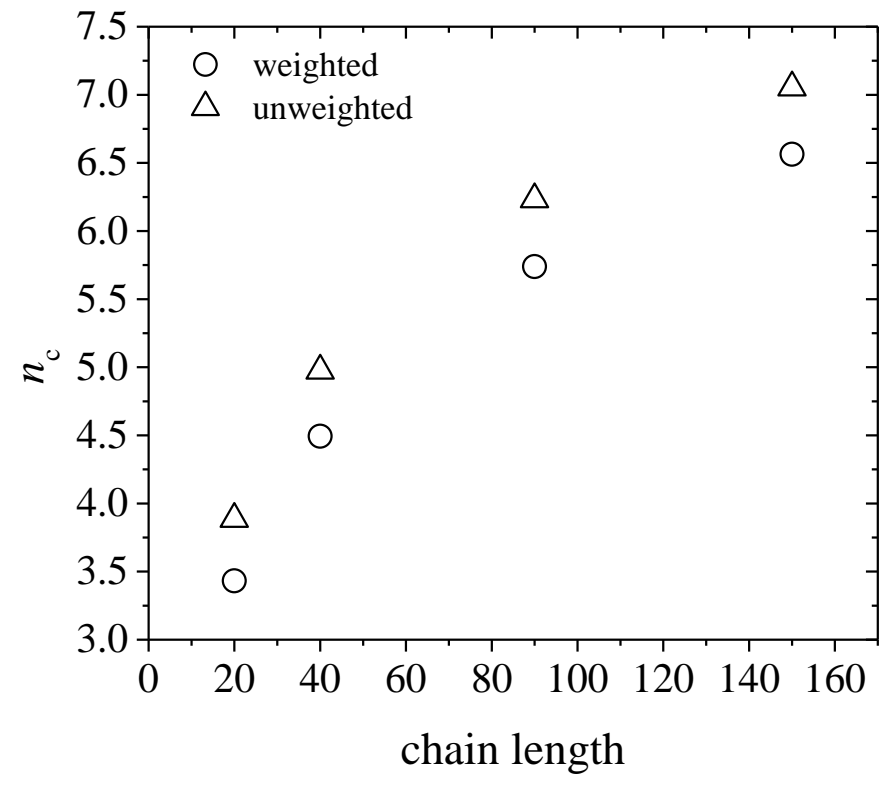

Figure S3.5. The effective conjugation length in units of consecutive planar angles $n_{\mathrm{c}}$ as computed from the corresponding weighted (circles) and unweighted (triangles) distributions with FF-6 $(T=700 \mathrm{~K}, P=1 \mathrm{~atm})$.

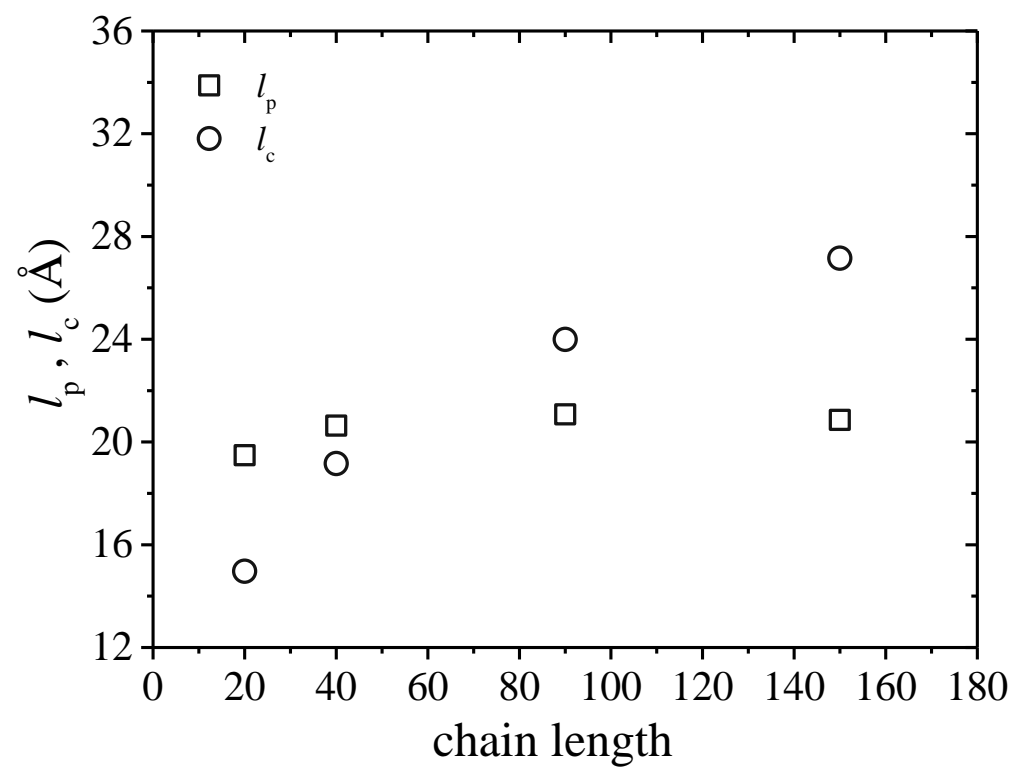

Figure S3.6. MD simulation predictions with FF-6 for the persistence length and the unweighted effective conjugation length of $n$-P3HT with $n=20,40,90$, and $150(T=700 \mathrm{~K}, P=1 \mathrm{~atm})$. The error bars are comparable to or smaller than the size of the symbols representing them and have been omitted for clarity. 
E. Estimation of the ECL based on the weighted $n_{\mathrm{c}}$ distributions for 20-P3HT and 90-P3HT
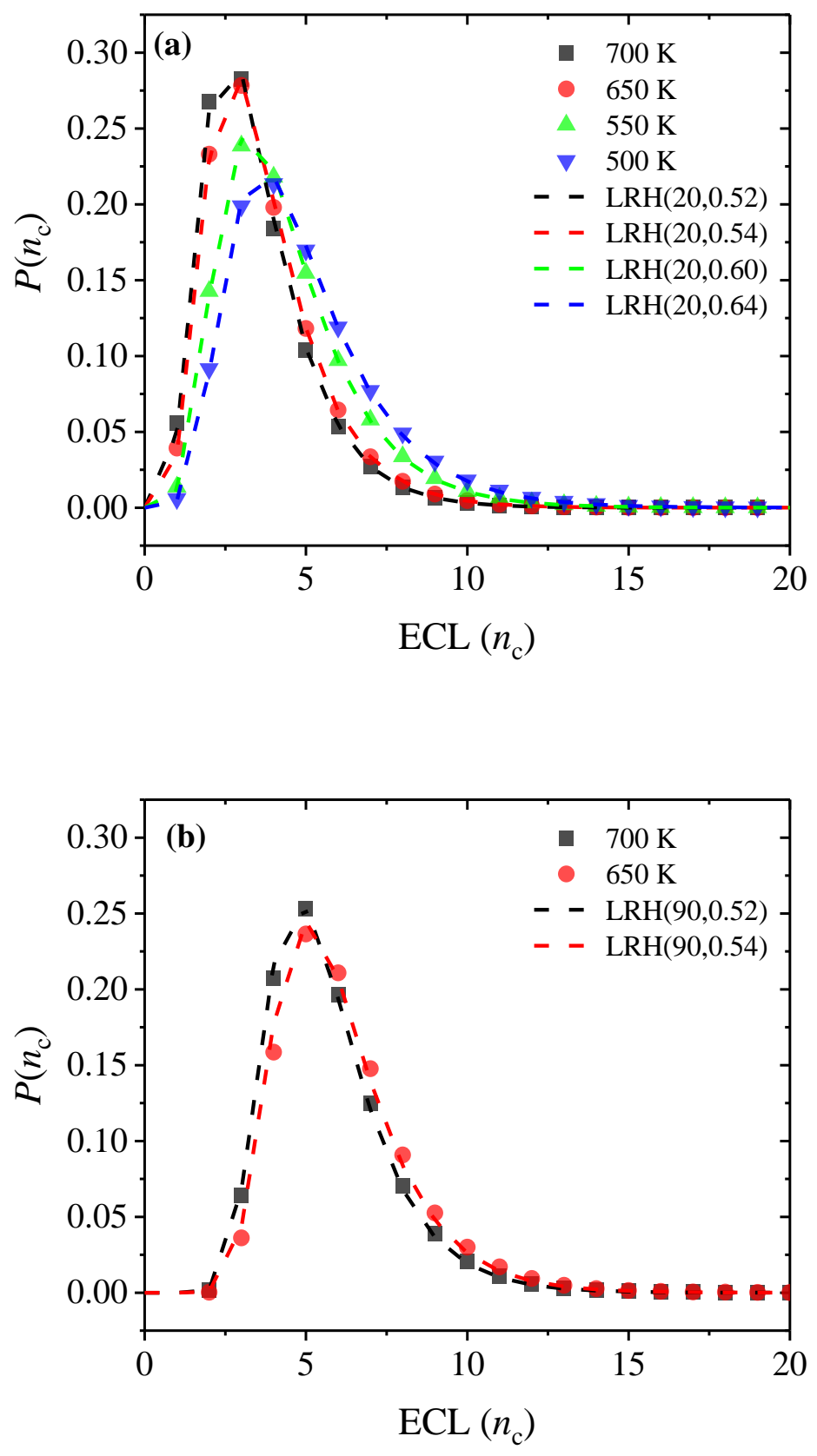

Figure S3.7. MD simulation predictions for the probability distribution of the effective conjugation length for: (a) the 20-P3HT, and (b) the 90-P3HT system. Results are shown from the simulations with FF-6 at different temperatures $(P=1 \mathrm{~atm})$. 


\section{References}

1. Van der Spoel, D.; Lindahl, E.; Hess, B.; Groenhof, G.; Mark, A.E.; Berendsen, H.J.C. GROMACS: Fast, flexible, and free. J. Comput. Chem. 2005, 26 (16), 1701-1718.

2. Poelking, C.; Andrienko, D. Effect of polymorphism, regioregularity and paracrystallinity on charge transport in poly(3-hexylthiophene) [P3HT] nanofibers. Macromolecules 2013, 46 (22), 8941-8956.

3. Cheung, D.L.; McMahon, D.P.; Troisi, A. Computational study of the structure and charge-transfer parameters in low-molecular-mass P3HT. J. Phys. Chem. B 2009, 113 (28), 9393-9401.

4. Huang, D.M.; Faller, R.; Do, K.; Moule, A.J. Coarse-grained computer simulations of polymer/fullerene bulk heterojunctions for organic photovoltaic applications. J. Chem. Theory Comput. 2010, 6 (2), 526-537.

5. Schwarz, K.N.; Kee, T.W.; Huang, D.M. Coarse-grained simulations of the solution-phase selfassembly of poly(3-hexylthiophene) nanostructures. Nanoscale 2013, 5 (5), 2017-2027.

6. Alberga, D.; Mangiatordi, G.F.; Torsi, L.; Lattanzi, G. Effects of annealing and residual solvents on amorphous P3HT and PBTTT films. J. Phys. Chem. C 2014, 118 (16), 8641-8655.

7. Moreno, M.; Casalegno, M.; Raos, G.; Meille, S.V.; Po, R. Molecular modeling of crystalline alkylthiophene oligomers and polymers. J. Phys. Chem. B 2010, 114 (4), 1591-1602.

8. Alexiadis, O.; Mavrantzas, V.G. All-atom molecular dynamics simulation of temperature effects on the structural, thermodynamic, and packing properties of the pure amorphous and pure crystalline phases of regioregular P3HT. Macromolecules 2013, 46 (6), 2450-2467.

9. Mayo, S.L.; Olafson, B.D.; Goddard, W.A. Dreiding - A generic force-field for molecular simulations. J. Phys. Chem. 1990, 94 (26), 8897-8909.

10. Jorgensen, W.L.; Tiradorives, J. The OPLS potential functions for proteins - Energy minimizations for crystals of cyclic peptides and crambin. J. Am. Chem. Soc. 1988, 110 (6), 1657-1666. 
11. Jorgensen, W.L.; Tirado-Rives, J. Molecular modeling of organic and biomolecular systems using BOSS and MCPRO. J. Comput. Chem. 2005, 26 (16), 1689-1700. 